Portland State University

PDXScholar

6-9-2016

\title{
The Investigation and Optimization of a Two-Heat- Pump System Incorporating Thermal Storage for Shaping Residential Heating Load
}

Emily Lord Barrett

Portland State University

Follow this and additional works at: https://pdxscholar.library.pdx.edu/open_access_etds

Part of the Power and Energy Commons

Let us know how access to this document benefits you.

Recommended Citation

Barrett, Emily Lord, "The Investigation and Optimization of a Two-Heat-Pump System Incorporating Thermal Storage for Shaping Residential Heating Load" (2016). Dissertations and Theses. Paper 3025. https://doi.org/10.15760/etd.3020

This Thesis is brought to you for free and open access. It has been accepted for inclusion in Dissertations and Theses by an authorized administrator of PDXScholar. Please contact us if we can make this document more accessible: pdxscholar@pdx.edu. 
The Investigation and Optimization of a Two-Heat-Pump System Incorporating Thermal

Storage for Shaping Residential Heating Load

by

Emily Lord Barrett

A thesis submitted in partial fulfillment of the requirements for the degree of

\author{
Master of Science \\ in \\ Electrical and Computer Engineering
}

Thesis Committee:

Robert Bass, Chair

Ross Gadient

Jonathan Bird

Huafen $\mathrm{Hu}$

Portland State University

2016 
(C) 2016 Emily Lord Barrett 


\begin{abstract}
Portland General Electric has proposed coupling one or more water tanks with two heat pumps in order to perform load-shifting in residential customer heating and cooling applications. By using the water tanks as a thermal storage unit, this project attempts to partially decouple energy consumption from generation to provide peak demand reduction and to better facilitate the integration of variable renewable energy resources. A scoping study was performed to evaluate the potential impact of this project if implemented in single family homes in Portland, Oregon. This study revealed that the system could provide meaningful savings in the cost of electricity to both the customer and utility. Additionally, an optimization algorithm was developed to dictate system operation and to maximize gains to the utility. Evolutionary algorithms were explored in an attempt to increase the effectiveness of the algorithm's search in limited computation time. Ultimately, an evolution strategy was selected as the most suitable based on tests run in winter and spring months. A genetic algorithm was then developed to handle fixed-speed heat pump operation for compatibility with an alpha-system prototype that has been developed by the research team.
\end{abstract}


Dedication

To Crystal Eppinger, who has made this last year make so much more sense. 


\section{Acknowledgements}

This project was wholly funded and motivated by Portland General Electric, without which this work would not have been conducted. In particular, I would like to gratefully acknowledge the support of Dr. Conrad Eustis, who not only conceived of the technological solution investigated here, but worked with the research team throughout this process and continually provided advice, insight and resources.

I would also like to acknowledge my past and current research teams, which have included Dr. Huafen Hu, Adam Henke, Timothy Gulzow, and Michael Davis, with a particular thank you to Kurtis Bleak. I have been very lucky to work with you all.

And finally, I would like to recognize the incredible impact that Dr. Robert Bass has had, both as the lead researcher on this research team and as my mentor, both and academic advisor. It is because of him that I not only worked on this project, but also decided to to return to get my Master's of Science in Electrical Engineering. 


\section{Contents}

$\begin{array}{ll}\text { Abstract } & \text { i }\end{array}$

Dedication $\quad$ ii

Acknowledgements

List of Tables $\quad$ vi

List of Figures $\quad$ vii

1 Introduction 1

1.1 Problem Statement . . . . . . . . . . . . . . . . . . . 1

1.2 Objective of the Project . . . . . . . . . . . . . . . . . 3

1.3 System Description . . . . . . . . . . . . . . . . . . 3

1.4 Related Research . . . . . . . . . . . . . . . . . . 5

1.4.1 Thermal Storage as a Distributed Energy Resource . . . . . . . . 5

1.4.2 Evolutionary Algorithms ................ 6

2 System Models $\quad 8$

2.1 Thermodynamic Models . . . . . . . . . . . . . . . . . 8

3 Scoping Study Design Methodology 11

3.1 Case Study . . . . . . . . . . . . . . . . . . . 11

3.1 .1 Weather Data . . . . . . . . . . . . . 14

3.1 .2 Pricing Information . . . . . . . . . . . . . . . . . . . . . . . . . . 14

3.1.3 Cost Assessment . . . . . . . . . . . . . . . . 15

3.2 Customer Heat Pump Operation _ . . . . . . . . . . . . . . . 16

3.3 Utility Heat Pump Operation . . . . . . . . . . . . . . . . . 17

3.4 Benchmark ........................... 20

3.5 Peak Demand Reduction . . . . . . . . . . . . . . . . . 22

4 Scoping Study Results $\quad 23$

5 Optimization Design $\quad 25$

5.1 Objective Function . . . . . . . . . . . . . . 25 
5.1 .1 Cost Function . . . . . . . . . . . . . . . . 27

5.1.2 System Constraints . . . . . . . . . . . . . . . 28

5.2 Challenges of Optimization . . . . . . . . . . . . . . 28

5.3 Evolutionary Computation . . . . . . . . . . . 30

5.3.1 Advantages . . . . . . . . . . . . . . 30

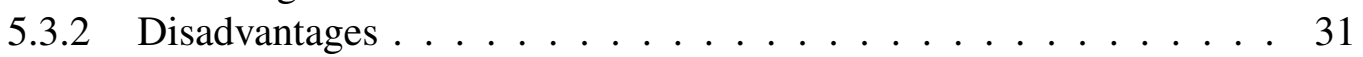

5.4 Algorithm Development . . . . . . . . . . . . . . . . 32

5.5 Evaluating Solution Fitness . . . . . . . . . . . . . . . . . . 32

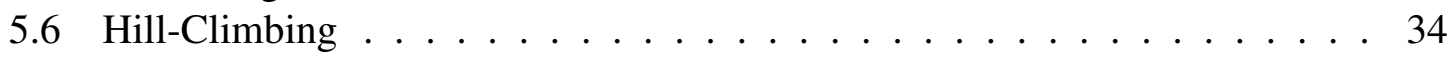

5.7 Evolution Strategy . . . . . . . . . . . . . . . . . . . . . . . . . . . . . . . .

5.8 Differential Evolution . . . . . . . . . . . . . . . . . 42

6 Optimization Results 46

7 Discussion $\quad \mathbf{5 1}$

7.1 Algorithm Performances . . . . . . . . . . . . . . . . 53

7.2 Limitations and Future Work . . . . . . . . . . . . . . . . . 54

8 Optimization for the Alpha System Prototype 57

8.1 Alpha Prototype . . . . . . . . . . . . . . . . . 57

8.2 Optimization for Discrete Heat Pump Operation . . . . . . . . . . . . . 58

9 Conclusion $\quad 62$

$\begin{array}{ll}\text { Bibliography } & 64\end{array}$

Appendix A: COP Equations 69

A.1 Variable Definitions . . . . . . . . . . . . . . . . 69

A.2 Benchmark Air-to-Air Heat Pump . . . . . . . . . . . . . . . . 69

A.3 Utility-Side Heat Pump . . . . . . . . . . . . . . . . . . 70

A.4 Customer Heat Pump Coefficient of Performance . . . . . . . . . . 70 


\section{List of Tables}

3.1 Benchmark Heating Results . . . . . . . . . . . . . . . . . . 22

4.1 Percent Cost and Energy Reduction for Heating . . . . . . . . . . . . . 23

4.2 Peak Reduction for 10,000 Homes . . . . . . . . . . . . . . . 23

5.1 Evolution Strategy Parameters . . . . . . . . . . . . . . . . . 41

5.2 Differential Evolution Parameters . . . . . . . . . . . . . . . . 45

6.1 Average Algorithm Improvement Over Iterative Method for January 2012 . . . 46

6.2 Standard Deviations for January 1, 2012 Over 100 Runs . . . . . . . . . . . . 46

6.3 Average Algorithm Improvement Over Iterative Method for April 2014 . . . . . 47

6.4 Algorithm Results from January 2012 in Dollars per Residence in Wholesale Electricity Costs . . . . . . . . . . . . . . . . . . . . . . . . . . 49

6.5 Algorithm Results from April 2014 in Dollars per Residence in Wholesale Electricity Costs . . . . . . . . . . . . . . . . . . . 50

8.1 Genetic Algorithm Parameters . . . . . . . . . . . . . . . . 60 


\section{List of Figures}

1.1 System Diagram . . . . . . . . . . . . . . . . . . . 4

3.1 Utility-Side Heat Pump COP Surface . . . . . . . . . . . . . . . . . . . 12

3.2 Customer-Side Heat Pump COP Surface . . . . . . . . . . . . . . . . . . 13

3.3 Flow Diagram Describing the Iterative Method to Obtain Heat Pump Operational Vector . . . . . . . . . . . . . . . . . . . . 19

3.4 Benchmark Air-to-Air Heat Pump COP Curve . . . . . . . . . . . . . . . . 21

5.1 Flow Diagram Describing the Hill-Climbing Process . . . . . . . . . . . . 36

5.2 Flow Diagram Describing the Evolution Strategy Process . . . . . . . . . . . 40

5.3 Flow Diagram Describing the Differential Evolution Process . . . . . . . . . . 44

7.1 PGE 2020 Q2 Supply and Demand for Flexibility . . . . . . . . . . . . . . 52

7.2 Improvement Seen in the Objective for Minimizing Utility Cost for Each Algorithm 55

8.1 Example Plot of Best Solution Evolution . . . . . . . . . . . . . . . 61 


\section{Introduction}

\subsection{Problem Statement}

Increases in peak power demand and the greater penetration of non-dispatchable generating plants on the electric power system present growing challenges to electric power providers. As the gap between peak and average electricity demand widens, the average utilization levels for generating plants decreases, increasing the cost associated with maintaining the capacity required to serve peak demand periods. The addition of more renewable energy plants such as wind power onto the grid introduces an increasing amount of intermittent generation. This creates a need for the ability to shift demand both to decrease peak periods and to better utilize non-dispatchable generating resources. Residential heating and cooling loads provide an opportunity to use thermal storage as a mechanism to shift power usage.

This project proposes the use of one or more water tanks as a thermal storage unit with two heat pumps each operating autonomously on the water tank. A larger, utility-operated air-to-water heat pump charges the thermal storage unit at low demand or high production times when the cost of wholesale electricity is low. A smaller, customer-owned heat pump then heats or cools the home using the water tank as a source. Utilizing the energy storage capability of the water tank improves the efficiency of the customer-side heat pump, thereby reducing customer cost and lowering power consumption during peak demand times. 
In order to realize these gains, an algorithm is required to determine an operational schedule that will optimize system performance. Optimal performance is defined as an operational schedule for the utility-side heat pump that minimizes some cost function. Within the context of this work, the objective considered will primarily be minimizing the cost of wholesale electricity to the utility; however, the minimization of customer cost, energy consumption, and peak power demand were also considered over the course of the project.

The optimization routine must be able to predictively determine an operational schedule for the utility-side heat pump over a rolling 24-hour period. Optimal performance will depend on a cost function that supplies cost weights to the system's power consumption over each time interval and the amount of power that each heat pump consumes. Power consumption is dictated by the set point and coefficient of performance (COP) of each heat pump. The COP of the utility-side heat pump is a function of outdoor temperature, which will also dictate the required set points for the customer-side heat pump to ensure customer comfort. In the case where utility cost is the objective function, the wholesale cost of electricity during each time interval will also dictate optimal performance. For the reduction of peak power consumption, the predicted period of peak consumption is required. The algorithm needs to be able to take in these driving data, as well as real-time sensor readings, and determine the optimal operation of the system over the next 24 hours. The algorithm will then run every hour, updating these set points on a rolling basis. It is therefore critical that the optimization routine be able to run consistently in less than an hour. 
Due to challenges in the optimization problem, including the size of the problem, the non-smooth objective function, non-continuous variable constraints and restrictions on the allowable algorithm run-time, stochastic methods were ultimately explored. This led to the development of two evolutionary algorithms, the more successful of which is an evolution strategy. Finally, a third algorithm was created to operate an alpha system prototype.

\subsection{Objective of the Project}

First, a scoping study was performed to assess the potential impact of the project. The goal of this study was to quantify potential benefits to both the utility and the customer in order to evaluate the project concept. Simulations were performed using generic models that provided estimates on the potential reduction in customer cost, wholesale electricity cost, peak demand and energy consumption. These models and results were then used in the development of an optimization algorithm capable of reliably determining an operational schedule for the utility-side heat pump to realize these system gains within the operational schedule of the system. Finally, a modified optimization routine was developed to operate a smaller, alpha system prototype using fixed speed heat pumps.

\subsection{System Description}

The purpose of the system is to provide greater load flexibility in residential heating and cooling applications by implementing a thermal storage unit between two heat pumps. An air-to-water heat pump is used to heat and cool the water tank. This heat pump would be 
owned and operated by the electric utility and used to charge the thermal storage unit during low consumption and over-generation periods. An air-to-water heat pump heats and cools the home within a range of temperatures set by the resident and would be owned by the customer. A diagram of the system configuration is given in Figure 1.1.

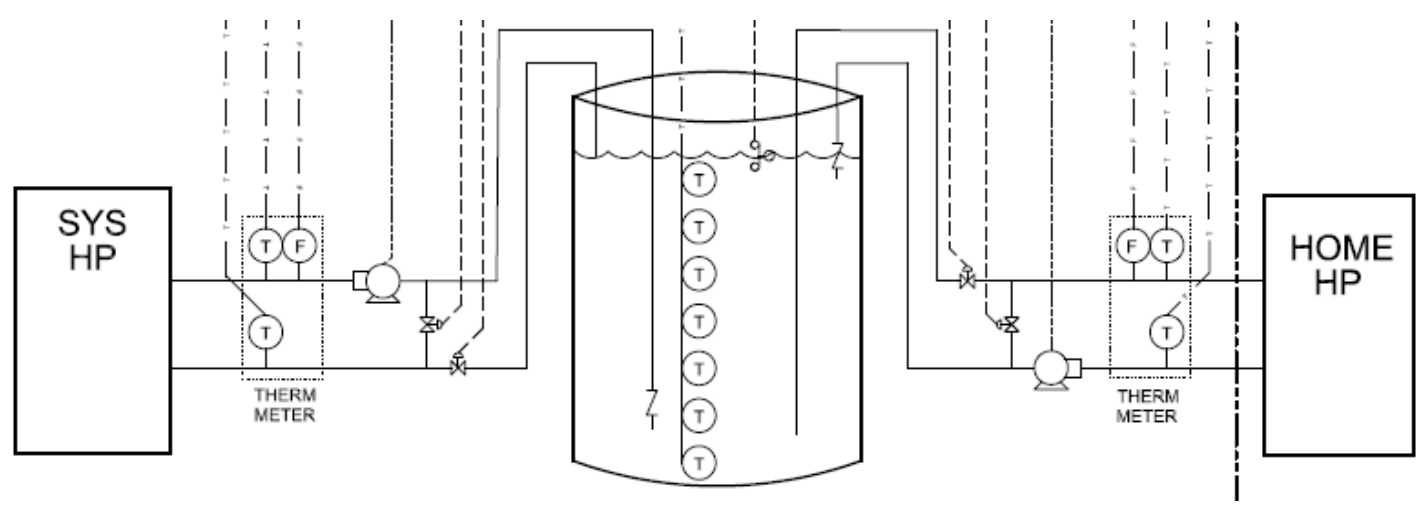

Figure 1.1: System Diagram

The customer-side heat pump will tend to have a higher efficiency than an air-to-air heat pump of the same size because it uses the water tank as a source rather than the ambient outdoor environment. Additionally, this efficiency improves further when the utility-side heat pump either pre-heats or pre-cools the tank as appropriate for the season. The water tank is therefore used as a thermal storage unit to partially decouple energy generation from end-user consumption.

Thermal energy extracted from the tank by the customer-side heat pump would be billed to the customer by the utility by the therm ${ }^{1}$. Based on preliminary discussion with Portland General Electric (PGE), this work assumes that therms are billed at a flat rate based on the average wholesale cost of electricity, the average coefficient of performance (COP) of the air-to-water heat pump and standard transmission and distribution fees.

\footnotetext{
${ }^{1} \mathrm{~A}$ therm is a unit used to measure heat energy and is equivalent to $100,000 \mathrm{BTU}$ or $1.055 \times 10^{8}$ joules.
} 


\subsection{Related Research}

\subsubsection{Thermal Storage as a Distributed Energy Resource}

Research on the use of distributed storage as a mechanism for shifting demand continues to generate a lot of interest, particularly as a mechanism for the better integration of renewable energy resources (1). The services that a storage unit can provide to the grid depend not only on its power capacity but also in large part on its energy capacity, and namely the length of time that it can supply its full power rating. Short duration storage units on the order of 3 to 20 minutes can operate as spinning reserves on the grid and provide smoothing for wind and compensate for intermittent reduction in photovoltaic production due to passing cloud-cover. However, to provide real peak-shaving, a full power duration of at least 2 hours is required (2). Within the context of a water tank coupled with a heat pump, the power capacity is dictated by the size of the heat pump while the energy capacity depends on the size of the thermal storage unit.

The use of hot water tanks with electric water heaters as thermal storage units has been widely explored as a mechanism for providing demand response (3), (4), (5). Because of the inertia inherent to a sufficiently large thermal mass, heating applications in general, and heat pump systems in particular, have been looked to as a mechanism for providing short term control of power consumption (6). Coupling a water tank with a heat pump has also been proposed as a strategy for reducing the peak power delivered to the grid by a local photovoltaic (PV) system, where the heat pump would charge the tank to store excess electricity production during times of high irradiance and then use the tank as a source for 
space heating during low PV production times (7).

\subsubsection{Evolutionary Algorithms}

The bases for the first three primary forms of evolutionary algorithm developed in parallel around the mid-1960s (8). The foundation for evolutionary programming was established by Lawrence Fogel in San Diego, California (9). Simultaneously, the groundwork for genetic algorithms were laid at the University of Michigan in Ann Arbor by John Holland (10).

Seminal work on the evolution strategy (ES) was done by a group of students in Berlin. Ingo Rechenberg purposed the use of random perturbations in physical parameters to find the optimal shapes of bodies in a flow, and in 1970 received his doctorate for his thesis, Optimization of Technical Systems According to the Principles of Biological Evolution (11), which describes a two-membered evolution strategy of the form $(1+1)$ and proposes the multimembered evolution strategy of the form $(\mu+1)$. Hans-Paul Schwefel, who was working with Rechenberg at the time, was the first to test the (1+1)-ES on a mechanical calculating machine (12). In his doctoral thesis, Evolutionary Strategy and Numerical Optimization, he presented his work on the $(\mu+\lambda)$-ES and $(\mu, \lambda)$-ES, which have since been widely adopted by the research community.

The development of differential evolution (DE) came later in the mid-1990s when Rainer Storn contacted Kenneth Price about using the genetic annealing algorithm that Price had published to solve the Chebyshev polynomial fitting problem. Through this attempt, Price began to work with arithmetic operations and eventually discovered the differential mutation operator that constitutes the foundation of DE. In 1995, Rainer and Price presented their 
preliminary results in a technical report entitled Differential Evolution - A Simple and Efficient Adaptive Scheme for Global Optimization (13), (14).

A selection of references for research related specifically to the use of evolutionary algorithms in optimizing scheduling and dispatch problems in power system applications is presented later in Section 5.3 within the context of the developing the optimization routine. 


\section{System Models}

\subsection{Thermodynamic Models}

The proposed system is comprised of an insulated, rectangular volume of air representing the home, an insulated, cylindrical water tank as a thermal storage unit, and two heat pumps: an air-to-water heat pump and a water-to-air heat pump, each coupled to the thermal storage unit. Equation 2.1 was taken from the Mathworks documentation on thermal modeling in Simulink (15) and was used to model the behavior of the home. Stratification was modeled within the insulated water tank based on work done by Angrisani et al (16). Equations 2.2 describe the the thermal behavior of the water tank and were derived from this work.

$$
\begin{gathered}
\dot{T}_{H}=\frac{\dot{Q_{h p}}+U A_{h}\left(T_{o}-T_{H}\right)}{m_{a} c_{a}} \\
\dot{T}_{1}=\frac{\left[k_{w} / \Delta x_{1}\right] A_{C}\left(T_{2}-T_{1}\right)+U_{l} A_{C}\left(T_{o}-T_{1}\right)+U_{t} A_{S}\left(T_{o}-T_{1}\right)+\dot{m}_{2} c_{w}\left(T_{2}-T_{1}\right)+\dot{m}_{1} c_{w}\left(T_{i n 1}-T_{1}\right)}{m_{w} c_{w}} \\
\dot{T}_{i}=\frac{\left[k_{w} / \Delta x_{i}\right] A_{C}\left(T_{i+1}+T_{i-1}-2 T_{i}\right)+U_{t} A_{S}\left(T_{o}-T_{i}\right)+\dot{m}_{1} c_{w}\left(T_{i-1}-T_{i}\right)+\dot{m}_{2} c_{w}\left(T_{i+1}-T_{i}\right)}{m_{w} c_{w}} \\
\dot{T}_{n}=\frac{\left[k_{w} / \Delta x_{n}\right] A_{C}\left(T_{n-1}-T_{n}\right)+U_{b} A_{C}\left(T_{o}-T_{n}\right)+U_{t} A_{S}\left(T_{o}-T_{n}\right)+\dot{m}_{1} c_{w}\left(T_{n-1}-T_{n}\right)+\dot{m}_{2} c_{w}\left(T_{i n 2}-T_{n}\right)}{m_{w} c_{w}}
\end{gathered}
$$




$$
\begin{aligned}
& T_{i n 1}=\frac{Q_{h p 1}}{\dot{m}_{1} c}+T_{n} \\
& T_{i n 2}=\frac{Q_{h p 2}}{\dot{m}_{2} c}+T_{1}
\end{aligned}
$$

$T_{H}$ is the indoor temperature, $T_{o}$ is the outdoor temperature, $U A_{h}$ is the equivalent thermal admittance of the home and $m_{a}$ and $c_{a}$ represent the mass and specific heat of the air in the home. $Q_{h p}$ is the heat transfer of the heat pumps, and in Equation 2.1, this always represents the heat transferred to or from the home by the customer-side heat pump.

$T_{1}, T_{i}$ and $T_{n}$ represent the water temperature at different layers in the tank where there are $n$ layers of stratification. The first layer is at the top, the $n$th layer is at the bottom of the tank and $i$ is every layer from 2 through $n-1 . T_{i n 1}$ and $T_{i n 2}$ are the temperatures of the water entering at the top and bottom layers respectively. This is dictated by the heat transfer of the two coupled heat pumps, the mass flow rate through the heat pumps and the water temperature entering the heat pumps. In heating mode, where the customer-side heat pump is heating the home, $T_{i n 1}$ represents the water temperature leaving the utility-side heat pump and $T_{i n 2}$ is the water temperature leaving the customer-side heat pump. In cooling mode, this is reversed. When heat is extracted from the tank, the corresponding $Q_{h p}$ becomes negative.

$A_{c}$ is the cross-sectional area of the tank, and therefore the area between stratified layers. $A_{s}$ is the surface area of the sides of the tank. Because the mass of the stratified layers are assumed to be the equal, this is equal to $\frac{1}{n}$ of the total surface area of the sides for 
each layer. The mass, specific heat and thermal conductivity of the water are given by $m_{w}$, $c_{w}$ and $k_{w}$ respectively. $\Delta x$ is the distance between the top and bottom of each layer of thermal stratification. The mass flow rates from the heat pumps are represented as $\dot{m}_{1}$ and $\dot{m}_{2}$ respectively, where $\dot{m}_{1}$ is flow injected at the bottom of the tank and removed at the top and $\dot{m}_{2}$ is flow in the opposite direction. 


\section{Scoping Study Design Methodology}

\subsection{Case Study}

The impact of the project was evaluated based on implementation in Portland, Oregon. Simulations were performed in Matlab assuming a 2,000 square foot, single family home with a thermal admittance (UA) of $400 \mathrm{kBtu} / \mathrm{hr}{ }^{\circ} \mathrm{F}$ (or $210 \mathrm{~W} / \mathrm{K}$ ) and $15 \%$ duct loss. These values were supplied by Portland General Electric as typical of single family homes in Portland where the system might ultimately be implemented. The thermal mass was taken to be a 600 gallon (or $2.3 \mathrm{~m}^{3}$ ) cylindrical tank of water with inlets and outlets at the top and bottom for each of the heat pumps. The utility-side heat pump and thermal mass were assumed to be installed outdoors with the water tank having an insulation of R-16. Thermal stratification was assumed to exist in the tank and was modeled in four layers.

The heat pumps were modeled using the coefficient of performance (COP) specifications of an air-to-water heat pump and a water-to-air heat pump respectively. Each heat pump therefore has its own set of COP curves, which depend on different operating conditions. The first COP equation described here represents a surface that was derived from the manufacturer specifications for a 3.2 ton Daikin air-to-water heat pump and is used to model the performance of the utility-side heat pump. The COP of this heat pump is a function of both the ambient air temperature and the water temperature. The surface was therefore 
created using a quadratic polynomial fitting function across the ambient air temperature and then across the water temperature values. The COP surface is given in Figure 3.1 and the equation is provided in Appendix A.

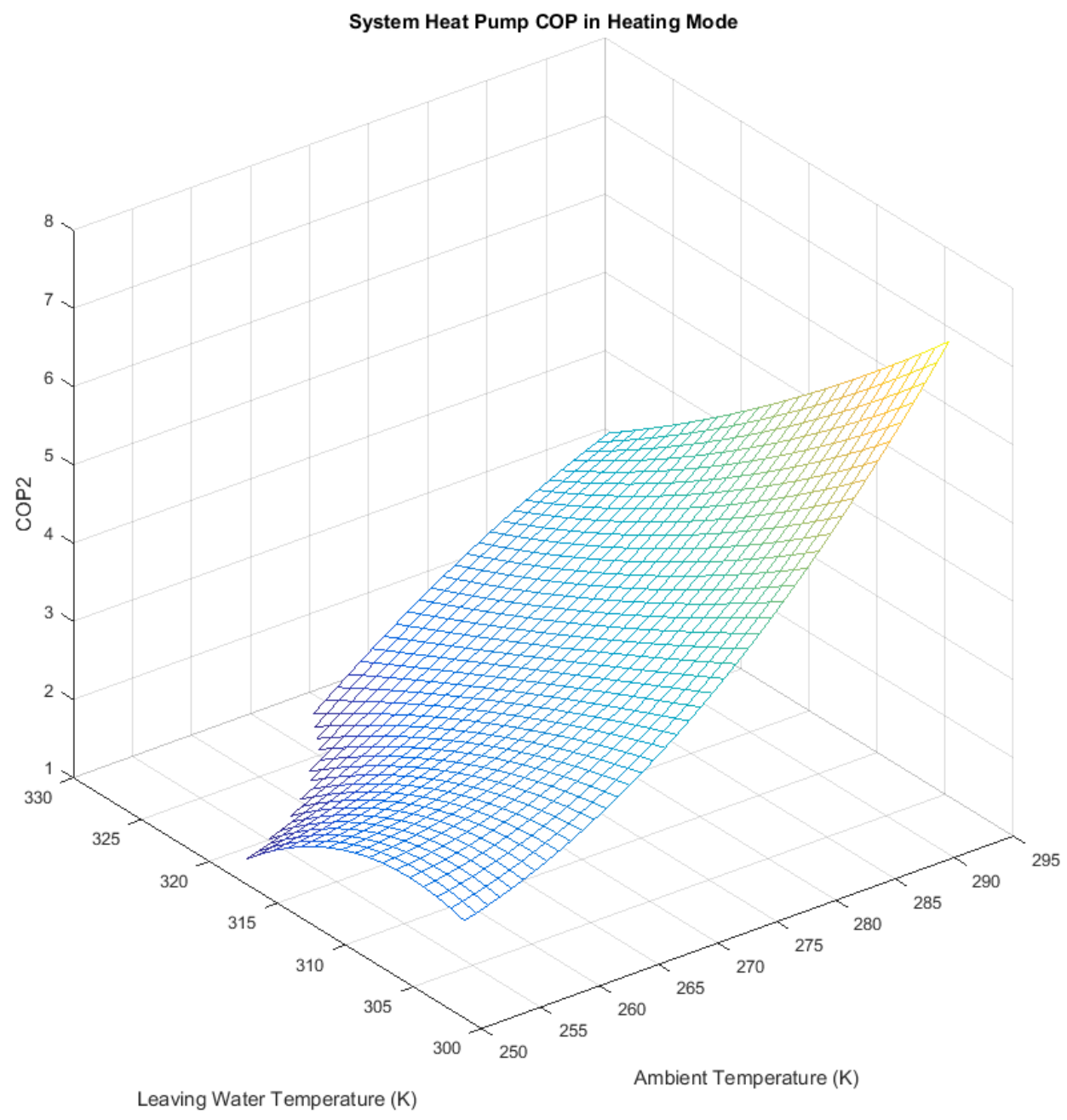

Figure 3.1: Utility-Side Heat Pump COP Surface

The customer-side water-to-air heat pump was modeled using the specifications of a 2 ton Trane water-to-air heat pump, and its COP is a function of the entering water temperature and the flow rate of the water. Again, the COP surface was derived by using a quadratic 
polynomial curve fitting function, this time across the water temperatures and then the flow rate. The COP surface for the customer-sized heat pump is given in Figure 3.2 and the equation is given in Appendix A. Water flow through the both of the heat pumps was assumed to be $1.5 \mathrm{gpm}$.

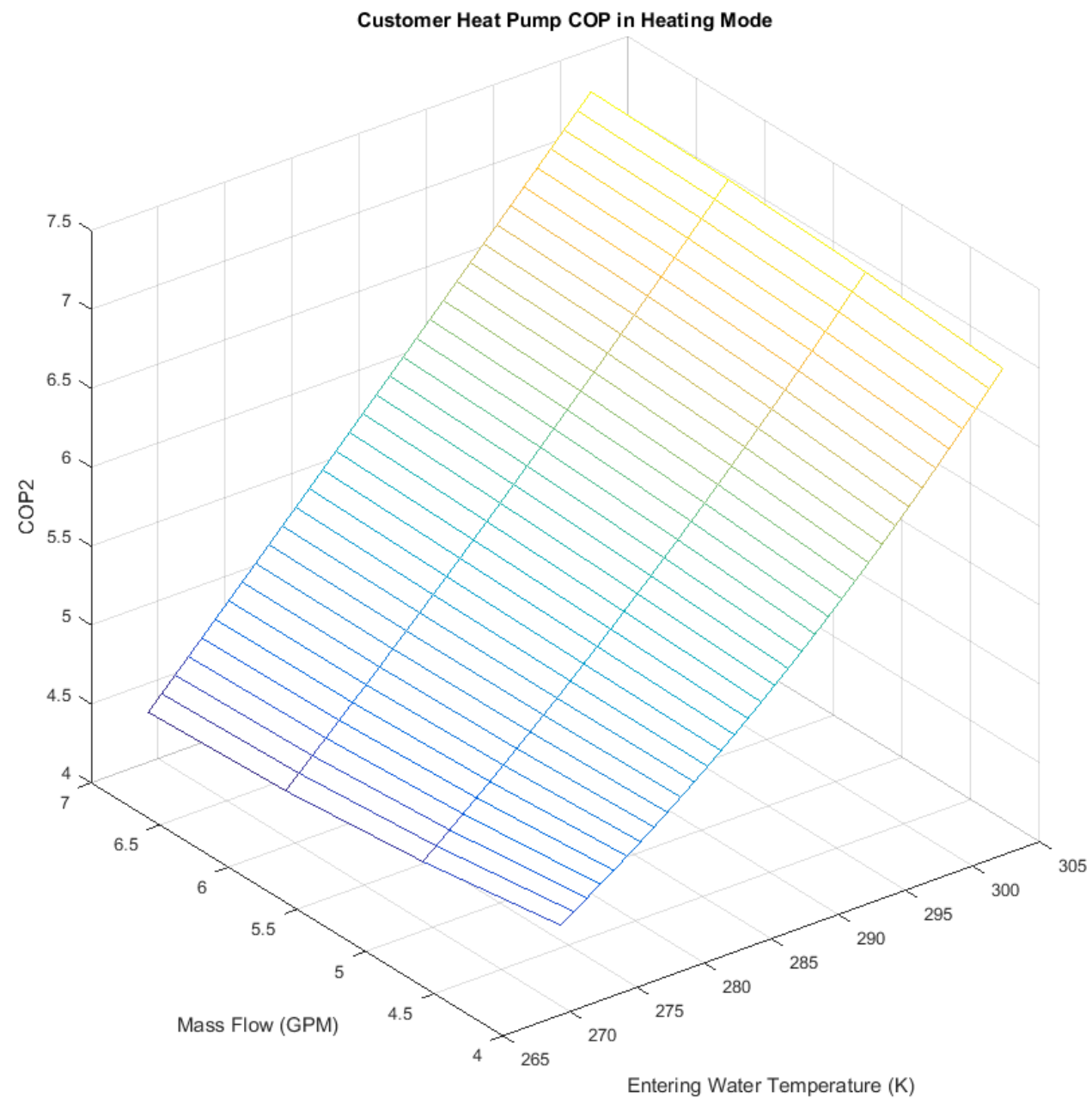

Figure 3.2: Customer-Side Heat Pump COP Surface 


\subsubsection{Weather Data}

Ambient temperature data typical for Portland Oregon were taken from the typical meteo-

rological year (TMY) database, developed by the National Renewable Energy Laboratory (NREL) (17). When evaluating the annual cost savings based on the daily performance of the system, each season was set up using the hourly temperature data typical for each day. For the peak demand reduction study, actual weather data were used from the days that saw the highest annual peak demand in 2013-2014, which was December 9th, 2013 for the winter peak. These data were taken from Weather Underground's historical database (18).

\subsubsection{Pricing Information}

The wholesale cost of electricity was derived from the locational marginal price (LMP) of electricity at the Malin hub of the California Independent System Operator (CAISO). The average hourly data from 2012-2014 were used to run three years of daily performance simulations. The retail cost of electricity to the customer is defined by Portland General Electric's (PGE) 2015 residential service flat rate schedule. The price of the thermal energy extracted by the customer-side heat pump from the thermal storage unit is based on the average wholesale cost of electricity at the Mid-Columbia hub in 2014, plus PGE's retail transmission and distribution rates, divided by the average $\mathrm{COP}$ of an air-to-water heat pump in the Portland area. This was calculated to be $\$ 0.022$ per therm. 


\subsubsection{Cost Assessment}

The cost of electricity for the utility is determined by the wholesale cost of all the electrical energy used by the heating system. For the two heat pump system, this is calculated by summing the power consumed by both heat pumps multiplied by the time interval and by the wholesale cost of electricity during each time interval for every time interval over the 24-hour period. This is given mathematically in Equations 3.1-3.3. For this work, this is done in intervals of 5 minutes. Five minutes was selected because it is the shortest control time interval possible given cycling limitations of the heat pumps.

$$
\begin{gathered}
J_{u t i l}=\sum_{n=1}^{288}\left(P_{1, n}+P_{2, n}\right) \Delta t C_{w h, n} \\
P_{1}=\frac{\dot{Q}_{1}}{C O P_{1}\left(T_{w 1}, T_{e n v}\right)} \\
P_{2}=\frac{\dot{Q}_{2}}{C O P_{2}\left(T_{w 2}, \dot{m}_{2}\right)}
\end{gathered}
$$

Where $J_{u t i l}$ is the wholesale cost of electricity required to operate the system in dollars, $n$ is each time interval, $P_{1}$ is the power consumed by the utility heat pump in kilowatts, $P_{2}$ is the power consumed by the customer heat pump in kilowatts, $\Delta t$ is the time in each interval in seconds, $C_{w h} t$ is the wholesale cost of electricity during each time interval in dollars, $Q_{1}$ is the rate of heat transfer in kilowatts from the utility side heat pump into the water tank, and $Q_{2}$ is the rate of heat transfer from the customer heat pump into the home. $C O P_{1}$ and 
$\mathrm{COP}_{2}$ are the COPs of the utility and customer heat pumps respectively as described in Section 3.1.

In the two heat pump system under investigation, customer cost is based on the cost of the energy consumed by the customer-side heat pump. This includes both the retail cost of the electrical energy required to run the heat pump as well as the thermal energy extracted from the thermal storage unit. Equation 3.4 describes how this was calculated.

$$
J_{\text {cust }}=\sum_{n=1}^{288}\left(P_{2, n}\right) \Delta t C_{r e t}+\Delta T \dot{m}_{2} c_{w} \Delta t C_{t h}
$$

Where $J_{\text {cust }}$ is the cost of energy to the customer in dollars, $C_{\text {ret }}$ is the retail cost of electricity, $\Delta T$ is temperature differential created across the inlet and outlet of the water loop passing through the customer heat pump as it operates in Kelvin, $\dot{m}_{2}$ is the mass flow rate of water through the heat pump in kilograms per second, $c_{w}$ is the specific heat of water in joules per kilogram-Kelvin, and $C_{t h}$ is the cost of thermal energy in dollars.

\subsection{Customer Heat Pump Operation}

The heating needs of the customer residence were provided by the customer-side heat pump using the thermal storage unit as a source. The temperature set point for the customer residence was taken from ASHRAE Standard 55 - Thermal Environmental Conditions for Human Occupancy, which recommends $71^{\circ} \mathrm{F}$ for the heating season with a swing tolerance of $+/-2{ }^{\circ} \mathrm{F}$. Additionally, a $2{ }^{\circ} \mathrm{F}$ set back was used from the hours of 9 am to 4 pm and from $11 \mathrm{pm}$ to 6 am. Heating in the home was simulated using the thermal home model described 
in Section 2.1, Equation 2.1, where the operation of the customer heat pump dictates $\dot{Q_{h p}}$ in each time interval. Heat pump operation was simulated in 5-minute increments where the pump was set to operate if the temperature fell to $2{ }^{\circ} \mathrm{F}$ below the set point and reheat the home up to but no higher than $2{ }^{\circ} \mathrm{F}$ above the set point. In this way, an operational vector for the customer-side heat pump was generated for the entire 24-hour period. When the simulations were initialized for the first day in the first month, the home was assumed to be exactly at the temperature set point.

\subsection{Utility Heat Pump Operation}

A method was developed to generate a vector of operational set points for the utility-side heat pump that maintains system constraints while setting the periods of operation to times of lower cost. This was then used to perform the scoping study. While this method provides a good, feasible solution to the problem of determining heat pump operation, it does not incorporate any of the optimization methods discussed later. However, because it is capable of producing low-cost, feasible solutions, it is also later used to seed and benchmark the optimization algorithms.

The routine assumes that the customer-side heat pump will operate autonomously to heat or cool the home within the prescribed comfort band agnostic of the wholesale cost of electricity. The utility heat pump is then set to operate during the lowest cost period of time as long as the total amount of energy extracted by the customer-side heat pump is still greater than the amount injected by the utility-side heat pump. The predicted temperatures 
for the water tank throughout the day are then found using these two preliminary operational vectors. The temperature of the water at the inlet to the utility heat pump is tested and the utility-side heat pump is triggered to operate based on a threshold temperature relative to the system's temperature constraints. This process is then iterated as described below.

This temperature threshold is varied between -10 and $10 \mathrm{~K}$ in steps of $1 \mathrm{~K}$, whereby the utility-side heat pump will be operated if the inlet water temperature is within this threshold of the minimum allowable tank temperature. System constraints are tested, and the routine goes through six iterations of adjusting the operational vector to correct any violations. If there are still constraint violations, the vector is rejected. Otherwise, the operational vector is saved. Once all 21 threshold temperatures have been tested, the cost of each of the resulting feasible solutions is found and then lowest cost solution is selected. Figure 3.3 illustrates this iterative method in a flow diagram.

The minimum tank temperature is set as $40^{\circ} \mathrm{F}$ and the maximum as $100{ }^{\circ} \mathrm{F}$, or $278 \mathrm{~K}$ and $311 \mathrm{~K}$ respectively, to accommodate the desired operational region of the heat pumps. When the simulations are initialized for the first day in the first month, the tank temperature is assumed to be $4 \mathrm{~K}$ above the minimum allowable tank temperature, or $282 \mathrm{~K}$. 
Figure 3.3: Flow Diagram Describing the Iterative Method to Obtain Heat Pump Operational Vector

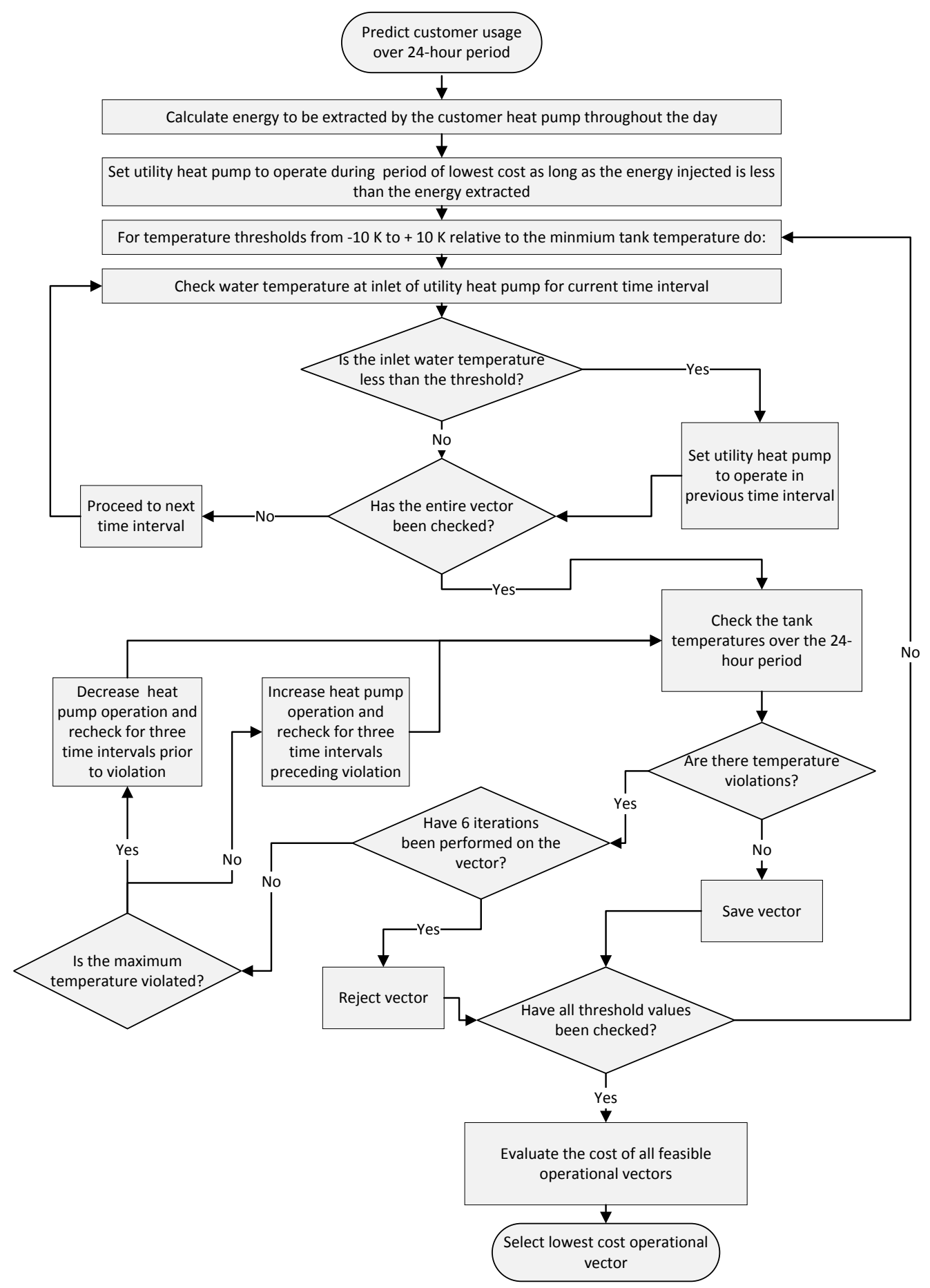




\subsection{Benchmark}

Simulations were run to develop a baseline with which to compare the results of the system study. For the benchmark, the same weather data, and wholesale and retail electricity prices are used as described in Sections 3.1.1-3.1.3. Equations 3.5-3.6 give the calculations performed to determine the cost to utility and customer respectively to operate the system.

$$
\begin{gathered}
J_{\text {utilbench }}=\sum_{n=1}^{288} P_{b, n} \Delta t C_{w h, n} \\
J_{\text {custbench }}=\sum_{n=1}^{288} P_{b, n} \Delta t C_{\text {ret }} \\
P_{b}=\frac{\dot{Q}_{b}}{\operatorname{COP}_{b}\left(T_{\text {env }}\right)}
\end{gathered}
$$

Where $J_{\text {utilbench }}$ is the cost to the utility of operating the benchmark system in dollars, $P_{b}$ is the power consumed by the benchmark air-to-air heat pump in kilowatts, $J_{\text {custbench }}$ is the cost to the customer for heating in dollars, $\dot{Q}_{b}$ is the rate of heat transfer from the air-to-air heat pump into the home and $\mathrm{COP}_{b}$ is the $\mathrm{COP}$ of the benchmark heat pump as described below and given in Appendix A.

The same thermal model for the home is used as described in Section 2.1. The operation of the heat pump is assumed to be identical to that of the customer-side heat pump described in Section 3.2, serving the same heating load based on the same losses and the same temperature set point of $71^{\circ} \mathrm{F}$ with a swing tolerance of $+/-2^{\circ} \mathrm{F}$. Heat pump operation is again simulated in 5-minute increments over a 24-hour period. The difference between the 
customer-side heat pump in the system under investigation and the benchmark is the type of heat pump serving the heating load, which is a 2-ton air-to-air heat pump rather than the 2-ton water-to-air heat pump described in Section 3.1. For the benchmark, heating is provided by a 2 ton air-to-air heat pump, which is modeled based on the specifications of a Carrier Home Comfort heat pump with a HSPF of 8.5. The COP curve for the Carrier Home Comfort unit is given in Figure 3.4 and the equation for calculating the COP is provided in Appendix A. Results per household for the benchmark simulations are presented in Table 3.1.

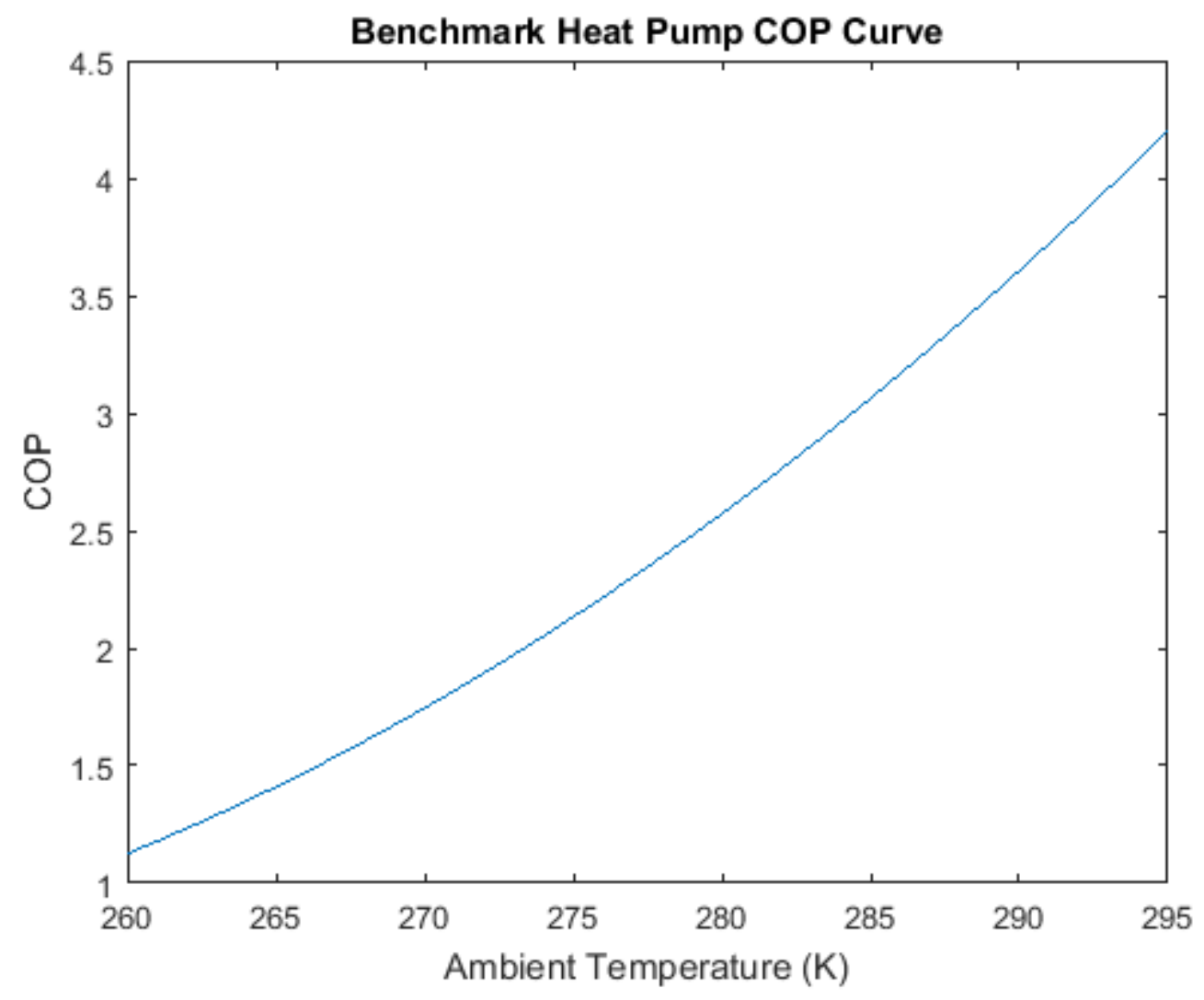

Figure 3.4: Benchmark Air-to-Air Heat Pump COP Curve 
Table 3.1: Benchmark Heating Results

\begin{tabular}{|c|c|c|c|}
\hline Season & Customer Cost & Utility Cost & Energy Usage \\
\hline Winter & $\$ 380$ & $\$ 120$ & $3,300 \mathrm{kWh}$ \\
\hline Spring & $\$ 170$ & $\$ 38$ & $1,500 \mathrm{kWh}$ \\
\hline Autumn & $\$ 250$ & $\$ 77$ & $2,200 \mathrm{kWh}$ \\
\hline Total & $\$ 800$ & $\$ 235$ & $7,000 \mathrm{kWh}$ \\
\hline
\end{tabular}

\subsection{Peak Demand Reduction}

According to data provided by PGE, the highest annual peak winter demand from 2013-2014 in their service territory occurred on December 9th, 2013 from 6pm-8pm. Ambient air temperatures were taken from Weather Underground (18), which gives the air temperature readings at the Portland International Airport (PDX) as $28.9^{\circ} \mathrm{F}$, or $271 \mathrm{~K}$, for each hourly reading from 2:53 pm until 8:53 pm. Operating at this outdoor temperature, the benchmark air-to-air heat pump would have a COP of 1.8. The ability of the two-heat-pump system to perform peak-shaving therefore depends on the difference in the maximum power consumption between this benchmark system and the water-to-air heat pump operating with the pre-charged water tank as a source over that peak demand period. This was determined by simulating the behavior of the air-to-water heat pump to meet heating demand based on the ambient air temperature during the peak period and assuming that the water tank had been preheated to the maximum allowable tank temperature. This analysis was then redone based on the manufacturer specifications for the water-to-air heat pump at the highest rated input water temperature, which is $85^{\circ} \mathrm{F}$. This case study was performed to address the uncertainty introduced by extrapolating the manufacturer data beyond the empirical data provided in the unit's specifications. Results for this work are given in Section 4. 


\section{Scoping Study Results}

Results from the scoping study are given in Tables 4.1-4.2. They demonstrate the potential for meaningful reduction in the cost of electricity to both the utility and to the customer as well as an overall reduction in energy consumption. Additionally, the system is capable of performing demand reduction during a two hour winter peaking event, which is of high value to the utility if the systems are aggregated.

Table 4.1: Percent Cost and Energy Reduction for Heating

\begin{tabular}{|c|c|c|c|}
\hline Season & Customer Cost & Utility Cost & Energy Usage \\
\hline Winter & $29 \%$ & $17 \%$ & $12 \%$ \\
\hline Spring & $24 \%$ & $13 \%$ & $7 \%$ \\
\hline Autumn & $24 \%$ & $9 \%$ & $5 \%$ \\
\hline Total & $26 \%$ & $14 \%$ & $9 \%$ \\
\hline
\end{tabular}

Table 4.2: Peak Reduction for 10,000 Homes

\begin{tabular}{|c|c|c|c|}
\hline Season & Benchmark (kW) & $\begin{array}{c}\text { Optimized (kW) } \\
\text { with } 100{ }^{\circ} \text { F Water }\end{array}$ & $\begin{array}{l}\text { Optimized (kW) } \\
\text { with } 85^{\circ} \text { F Water }\end{array}$ \\
\hline Winter & 26,000 & 9,600 & 12,000 \\
\hline
\end{tabular}

Gains to the customer are realized through the improved COP of the customer heat pump using the thermal storage unit as a source. This also allows for the reduction in consumption during peak demand times, which can be achieved by pre-charging the thermal storage unit in anticipation of peak demand periods. This reduces the power consumption of the customer-side heat pump while providing the same space conditioning. Based on the desired 
operating temperature range for the water tank and heat pumps, initial results showed a $63 \%$ reduction in power consumption during winter peaking times over the benchmark heating system. However, this analysis relies on the extrapolation of the heat pump's manufacturer specifications to inlet water temperatures for which the unit is not rated and where empirical characterization data are not available. This analysis was therefore also performed using COP information at the highest inlet temperature available, which yielded a lower but still significant peak power reduction of roughly $53 \%$. In addition to the potential for peakshaving, utility costs are reduced through the reduction of energy consumption and the shift of electricity consumption to times of lower wholesale electricity costs.

A five-minute step time was used in this work because that is the shortest interval over which the heat pumps can be operated given their cycling limitations. Ten-minute intervals were tested over the month of January 2012 using the iterative method and compared to the system's performance where five-minute results. Using the ten-minute intervals yielded a 9.7\% increase in cost over the month, which preliminary results seem to confirm that the higher granularity of control made available by using five-minute intervals improves system performance. However, decreasing the number of heat pump cycles would increase the life-expectancy of the equipment and so there is an implicit trade-off between the desired level of control and operating the system in such a way that reduces wear and tear. 


\section{Optimization Design}

\subsection{Objective Function}

The purpose of the optimization algorithm is to minimize an objective function, which is constructed by taking the product of the energy consumed by the system and a cost function. This cost function dictates the objective of the optimization algorithm by supplying the weighted cost of energy consumption during each time interval within the optimized period. For the scoping study and the optimization presented here, the cost function is simply the wholesale cost of energy during each time interval. This means that for this structure, the objective function also provides the cost of electricity to operate the heat pump system to the utility over the period of interest. This form for the objective function was selected not only for its simplicity, but because it is consistent with the way that operational optimization is commonly framed for heating and cooling applications (19), (20), (21), (22). Equations

5.1-5.4 define the objective function.

$$
\begin{gathered}
\min \left(F\left(P_{1}, P_{2}, \text { Cost }\right)\right) \\
F\left(P_{1}, P_{2}, \text { Cost }\right)=\sum_{n=1}^{288}\left(P_{1, n}+P_{2, n}\right) \Delta t \text { Cost }_{n}
\end{gathered}
$$




$$
\begin{gathered}
P_{1}=\frac{\dot{Q}_{1}}{\operatorname{COP}_{1}\left(T_{w 1}, T_{e n v}\right)} \\
P_{2}=\frac{\dot{Q}_{2}}{\operatorname{COP}_{2}\left(T_{w 2}, \dot{m}_{2}\right)}
\end{gathered}
$$

Subject to:

$$
\begin{gathered}
\dot{Q}_{1}=0 \quad \text { or } \quad 0.2 \dot{Q}_{1 \max } \leq \dot{Q}_{1} \leq \dot{Q}_{1 \max } \\
\dot{Q}_{2}=0 \quad \text { or } \quad 0.2 \dot{Q}_{2 \max } \leq \dot{Q}_{1} \leq \dot{Q}_{2 \max } \\
T_{\min } \leq T_{w} \leq T_{\max }
\end{gathered}
$$

$P_{1}$ and $P_{2}$ are the power consumed by the utility and customer heat pumps respectively. Cost is the function that dictates the cost of energy during each time interval, $n$, which is $t$ seconds in length. The objective function is therefore the sum of the cost of each interval, $n$, where $n$ goes from 1 to 288 , for 5 minute intervals over 24 hours.

$\dot{Q}_{2}$ is the rate of heat transfer of the customer-side heat pump, which is dictated by customer heating/cooling needs. For the sake of simplicity, the customer-side heat pump is assumed to operate autonomously, and $\dot{Q}_{2}$ is not optimized by the algorithm. $\dot{Q}_{1}$ is therefore the only optimized variable in the objective function. Equations for the coefficients of 
performance for each heat pump, $C O P_{1}$ and $\mathrm{COP}_{2}$, are given in the appendix. Both are functions of the water temperature in the tank, $T_{w 1}$ and $T_{w 2} . T_{w 1}$ and $T_{w 2}$ may or may not be essentially equal depending on the state of the tank and whether meaningful stratification exists. The COP of the utility-side heat pump, $C O P_{1}$ is also a function of the outdoor air temperature, $T_{\text {env }}$ while the customer-side heat pump's $\mathrm{COP}, \mathrm{COP}_{2}$, is a function of the water flow rate, $\dot{m}_{2}$.

\subsubsection{Cost Function}

For the case where energy consumption is minimized, the cost function would simply be unity, and the objective function becomes the sum of the energy consumed by each heat pump over each time interval in the period of optimization. For the purposes of this work, we will consider the cost function to be the hourly average Locational Marginal Pricing (LMP) for wholesale electricity at the Malin node of the California Independent System Operator (CAISO), which is the node at the California-Oregon intertie. As such, the cost function will be constant over each hour interval. Wholesale electricity prices will reflect high demand and over-generation periods, where prices will respectively be driven up or may even go negative in the case of over-generation times. However, additional weights could be incorporated into the cost function to further encourage pre-charging in anticipation of peak demand periods, to prohibit the operation of the utility-side heat pump during these times or to assist in ramping events. 


\subsubsection{System Constraints}

The variable speed heat pump can operate continuously between its maximum capacity, $\dot{Q}_{1 \max }$, and twenty percent of its maximum capacity, or it can be off, where $\dot{Q}_{1}$ is zero. These constraints are dictated by the equipment itself. Similarly, if the customer-side heat pump is variable speed, it can be off, where $\dot{Q}_{2}$ is zero, or it can operate continuously between $20 \%$ of its maximum capacity and its maximum capacity. Additionally, there are minimum and maximum allowable temperatures within the tank, $T_{\min }$ and $T_{\max }$, which have been set to $280 \mathrm{~K}$ and $322 \mathrm{~K}$ respectively. These limits are dictated by the operational capacity of the heat pumps themselves.

\subsection{Challenges of Optimization}

A primary requirement for operating the system successfully is that the optimization algorithm run consistently in a short enough period to update the operational vector. These set points must be updated every hour, meaning that the routine must be able to run within that time frame. The algorithm will therefore need to be able to take in new temperature sensor readings, update projected weather and cost information, and then generate a new vector of operating set points for the utility-side heat pump in less than hour. This presented a challenge given the size of the search space and the fact that the objective function is not convex.

Using 5-minute operational intervals over 24 hours creates a 288-dimensional space. Additionally, there are circular dependencies within the objective function. Namely, the 
COPs of both heat pumps are functions of the water temperature in the tank, which is determined by the operational vector being optimized. There is also a high degree of temporal interdependence between the optimized vectors. Adjusting the value of one variable in the vector will significantly change the impact of adjusting another relative to the temporal proximity of the two set points.

Initially, sequential quadratic programming (SQP) was explored as an optimization strategy. However, this presented serious challenges, including the inability to reliably converge on a feasible solution and inconsistent or unacceptable algorithm run-times, which both create barriers to operating the system. This was partially addressed by seeding the routine with a known good, feasible solution, created using the strategy described in Section 3.3. However, while this improved the SQP algorithm's ability to consistently converge on a feasible solution, run-times were still highly inconsistent, and the results were not meaningfully different than those provided by simply running the seeding routine.

Beyond the size of the problem and the need for consistent, fast run-times, there are other characteristics intrinsic to the problem that make optimization methods requiring the gradient or higher derivatives difficult to implement. Primarily, the objective function is not in general continuous. Both the power consumption of the heat pumps and the average hourly LMP data as framed here are discrete functions, meaning the objective function is not differentiable. The discontinuity in the variable constraints are also difficult to handle.

In addition to highlighting all of these challenges, this initial process made it clear that finding the global optimal solution was actually of less importance than finding a good, 
feasible solution within the physical constraints of the problem as well as the constraints imposed by operating the system. This altered the approach to optimization, and stochastic, direct search methods were explored in an attempt to perform more effective exploration of the search space in less computation time. This led to an investigation of evolutionary algorithms as well as memetic algorithms, which couple an evolutionary technique with a local search strategy.

\subsection{Evolutionary Computation}

Evolutionary computation has been explored within the context of optimizing the operation of the electrical grid and power delivery for a number of applications. In developing an intelligent energy management system for the optimal short-term scheduling of energy resources, Hable et al. determined that evolutionary algorithms were the most suitable optimization method (23). Evolutionary programming (24), real-coded genetic algorithms (25), evolution strategies (26) and differential evolution (27) have all been investigated as strategies for solving the economic dispatch problem.

\subsubsection{Advantages}

There are several advantages to using an evolutionary algorithm, including the ability to handle a non-differentiable objective function and discontinuous variable constraints. Their broad applicability also allows the structure developed to be simply adapted to treat different objectives or to handle different physical parameters and constraints. By stochastically initializing a population of solutions and then evolving them according to their value, a 
greater search of the space can be accomplished in less time, which reduces the risk of settling on a local minimum near the seeded solution as was seen using SQP. Additionally, computation time can be reliably restricted by setting the termination criteria to a set number of algorithm generations. Finally, the conceptual simplicity of evolutionary algorithms is a benefit, particularly given that the algorithm will need to be maintained by future engineering students on the research team.

\subsubsection{Disadvantages}

Within the context of this problem, there is no guarantee that an evolutionary algorithm will yield an optimal solution. Furthermore, the stochastic nature of the search means that the results are generally not reproducible. In other words, the same evolutionary algorithm can be run twice on the same data and yield different results each time because the solution set is initialized randomly, the evolution of each individual solution occurs based on probability, and the operators that adapt the solution use randomly generated values. If the results of the algorithm vary significantly enough from run to run, this could create serious concerns about the reliability of the algorithm's performance. This can be mitigated somewhat by using a large population and by running an algorithm multiple times and taking the best solution generated, but this increases the computation time. To assess the consistency of the algorithms' results, each algorithm was run 100 times on the same data and the standard deviation between the results was taken. Given that the context of this project, getting a high-quality, feasible solution in an acceptable run-time is of higher value than complete reproducibility or absolute optimality. The stochastic nature of evolutionary computation 
was deemed acceptable, and a compromise was attempted between computation speed, solution quality and algorithm consistency.

\subsection{Algorithm Development}

For each of the evolutionary algorithms that were developed, there were parameters that needed to be set for the application including probabilities, population size and the number of generations, among others. While there are sometimes general guidelines or accepted practices for selecting these parameter values, they often need to be tuned empirically by testing the algorithm over a limited data set and limited number of generations. For this work, that was done using data for January, 2012. The month of January was selected because it contains both the typical winter week and the extreme winter week in the typical meteorological year data for Portland, Oregon (17). Once the algorithms had been developed

they were also tested on the shoulder weather months, April and October, where the April tests were of particular interest because April is in the second quarter where the integration of wind energy generation creates the largest challenges in the region due to the inability to curtail hydroectric production for environmental reasons during high wind periods.

\subsection{Evaluating Solution Fitness}

Within the context of evolutionary computation, minimization problems are often framed as the maximization of a solution's "fitness." However, this is inconvenient when the cost function can be driven negative, which can occur during over-generation periods. Therefore, 
rather than framing the function in terms of the maximization of fitness, the problem is framed in terms of the minimization of the objective plus a penalty function that is used to enforce system constraints. The penalty function increases the solution's value relative to the magnitude of its constraint violation, V. A weight was placed on V to change the relationship between the solution to the objective function, F, and the violation. Each value between 0.5-5 at 0.5 intervals were tested empirically by running the three algorithms explored for 10 generations each using each weight during the first day in January, 2012. The weight was set at 2 because it produced the best results for all three algorithms for that day. The function that was used to evaluate the solution's objective value is given in Equation 5.5 below.

$$
S=F+2 V
$$

Where $\mathrm{S}$ is the solution value being minimized, $\mathrm{F}$ is the objective function given in 5.1 and $\mathrm{V}$ is the solution's constraint violation. 


\subsection{Hill-Climbing}

Before building an evolutionary algorithm for this optimization problem, hill-climbing was looked at as a very simple stochastic strategy, primarily as a method of benchmarking other algorithms and as a mechanism for performing local search, which could be used to augment a better global search. To begin, a population of 100 individuals is initialized randomly within the search space. This is done by using a random generator to select values for each operational set point with uniform distribution between 0 and the maximum operational set point, $\dot{Q}_{1 \max }$. If the random number is less than or equal to $10 \%$, the value is forced to zero. If it is between $10 \%$ and $20 \%$, the value is forced to $20 \%$, thereby enforcing the discontinuous variable constraint. Additionally, one individual in the population is set to the vector developed through the method described in Section 3.3. This ensures that at least one individual in the population is feasible upon initialization.

The value of each individual is then evaluated based on the solution value function given in Section 5.5. For each solution, hill-climbing is then performed, where each element in the solution is perturbed randomly within a prescribed neighborhood. The best solution is then selected and the process repeats itself for 20 iterations. Figure 5.1 illustrates this hill-climbing method in a flow diagram.

Initially, a neighborhood was defined as $6.25 \%$ of the maximum allowable variable value. If the variable constraints were violated during this process, they were enforced by setting the value of the variable at the nearest allowable value. For instance, if the heat transfer rate, $\dot{Q}_{1}$, would have exceeded the maximum allowed value, it was set to $Q_{1 \max }$. If the value was 
less than zero, it was set to zero. Similarly for less than $10 \%$ and between $10 \%$ and $20 \%$.

The neighborhood was then expanded dynamically to facilitate a greater search early in the algorithm run and decreasing in size with each subsequent level of search. In this dynamically-adapted run, the neighborhood for local search is defined by Equation 5.6, which was developed to decrease the size of the neighborhood based on the current search iteration, g, from a relatively large search space of $25 \%$ of the maximum variable value to a much smaller neighborhood of $2.4 \%$ at the end.

$$
N=\frac{1}{2(g+1)}
$$

Where $\mathrm{N}$ is a percentage of $\dot{Q}_{1 \max }$ and $\mathrm{g}$ is the current search iteration.

Both hill-climbing methods were tested for the month of January using typical meteorological data for Portland, Oregon and Malin LMP cost information from 2012. They both showed very marginal improvement over the initial iteratively-generated operational vector described in Section 3.3. The static neighborhood of $6.25 \%$ of the maximum allowable value yielded an improvement of roughly $0.1 \%$ while the dynamically adapted neighborhood did slightly better with an improvement of about $0.4 \%$. 
Figure 5.1: Flow Diagram Describing the Hill-Climbing Process

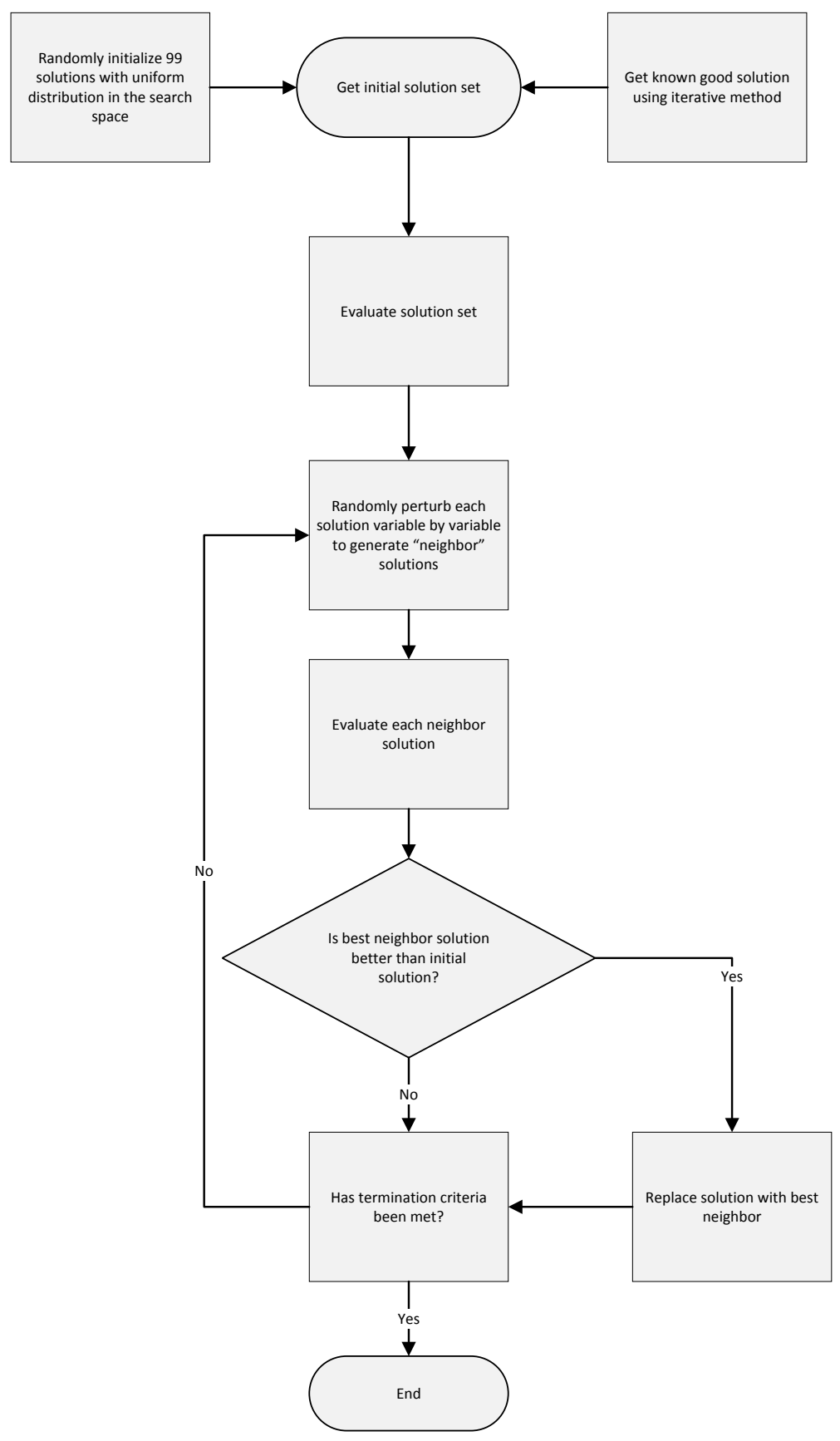




\subsection{Evolution Strategy}

The first evolutionary algorithm explored was a $(\mu / \rho, \lambda)$ evolution strategy (ES), which is a direct, stochastic search method that allows for the simultaneous adaption of the solution itself and the strategy parameter used to mutate each solution (28). The $(\mu / \rho, \lambda)$ form, as opposed to the $(\mu / \rho+\lambda)$ form, indicates that selection occurs from only the offspring population rather than the combined parent and offspring populations. The emphasis of an evolution strategy is on performing mutation on the solution rather than recombining solutions within the population. The structure used here is typical of a basic ES and involves the self-adaption of strategy parameters, the equations for which are given in 5.7-5.9 and were adopted from work done by H.P. Schwefel, (29), (12). This was initially chosen as an optimization strategy because the solutions themselves are encoded as real numbers and evolved as such, which is convenient within the context of this problem.

The algorithm begins by once again randomly initializing a population of real-numbered vectors using the same method as described in Section 5.6. The value of each solution is then immediately evaluated using the method described in Section 5.5 and the best solution is saved external to the algorithm. The highest quality solution is retained and updated as necessary to ensure it is not lost during evolution in the $(\mu / \rho, \lambda)-E S$ where the entire parent population is replaced by the next generation.

Vectors of strategy parameters must also be generated, which will then be evolved in parallel with the solutions (29). These were all initialized to one and then immediately adapted using Equation 5.7. 


$$
\sigma_{i j}^{\prime}=200 \sigma_{i j} \exp \left(\sqrt{(G+1-g)}\left(\tau^{\prime} N(0,1)+\tau N_{i}(0,1)\right)\right)
$$

Where $\mathrm{i}$ is the individual in the population whose corresponding strategy parameter is being adapted, $\mathrm{j}$ is the element in the strategy parameter vector being adapted, $\sigma$ is the strategy parameter prior to adaption, $\sigma^{\prime}$ is the new strategy parameter, $\mathrm{G}$ is the number of generations that the algorithm will be run for and $\mathrm{g}$ is the current generation. Equations for $\tau$ and $\tau^{\prime}$ are given in 5.8 and 5.9.

$$
\begin{gathered}
\tau=\frac{1}{\sqrt{2 \sqrt{k \mu}}} \\
\tau^{\prime}=\frac{1}{\sqrt{2 k \mu}}
\end{gathered}
$$

Where $\mathrm{k}$ is the number of elements in each vector, in this case 288 , and $\mu$ is the size of the parent population. The minimum value for $\sigma$ was set at $2 \%$ of the maximum allowable value for $\dot{Q}_{1}$. If sigma fell below this value, it was forced to $3 \%$ of $\dot{Q}_{1 \max }$. By making sigma a function of the current generation relative to the total number of generations the algorithm will run, the strategy parameters tend to decrease in magnitude as the algorithm progresses. This was done in an attempt to encourage global exploration early in the search process and then facilitate better exploitation of a local area as the algorithm reaches its conclusion.

Work was also done to evaluate the potential benefit of incorporating Rechenberg's 1/5 rule (12) to alter the size of the strategy parameters dynamically based on the percentage of successful mutations in the last set number of generations. The core concept here is to 
increase the size of the strategy parameters if mutation has successfully occurred on an individual greater than $20 \%$ of the time in the last several generations because the assumption is that the individual is not close to an optimal solution. Conversely, if mutation has had a less than $20 \%$ success rate, the strategy parameter value is decreased to increase the granularity of the search in that area under the assumption that the individual is converging on an optimal solution. However, during testing, this strategy did not increase the ability of the evolution strategy to find good solutions more quickly, which was attributed to the multi-modality of the fitness landscape.

Following the adaption of the strategy parameters, an offspring population is generated through mutation and recombination, where mutation occurs with a probably of $60 \%$. If mutation does not occur, two parent solutions are recombined to create an offspring. The recombination operator used is discrete recombination, also known as uniform cross-over, where elements are randomly selected with equal probability from each parent solution (30). The mutation operation is given in Equation 5.10 where $\mathrm{x}$ is the element undergoing mutation and $x^{\prime}$ is the result of mutation (12).

$$
x_{i j}^{\prime}=x_{i j}+\sigma_{i j} N(0,1)
$$

The constraints on $\dot{Q}_{1}$ are once again enforced during mutation using the same strategy described in Section 5.6. Each solution in the offspring population is then evaluated and hill-climbing is performed on every individual in the offspring pool with a search depth of 1 . Finally, a new parent population is selected deterministically based on the values of each 
Figure 5.2: Flow Diagram Describing the Evolution Strategy Process

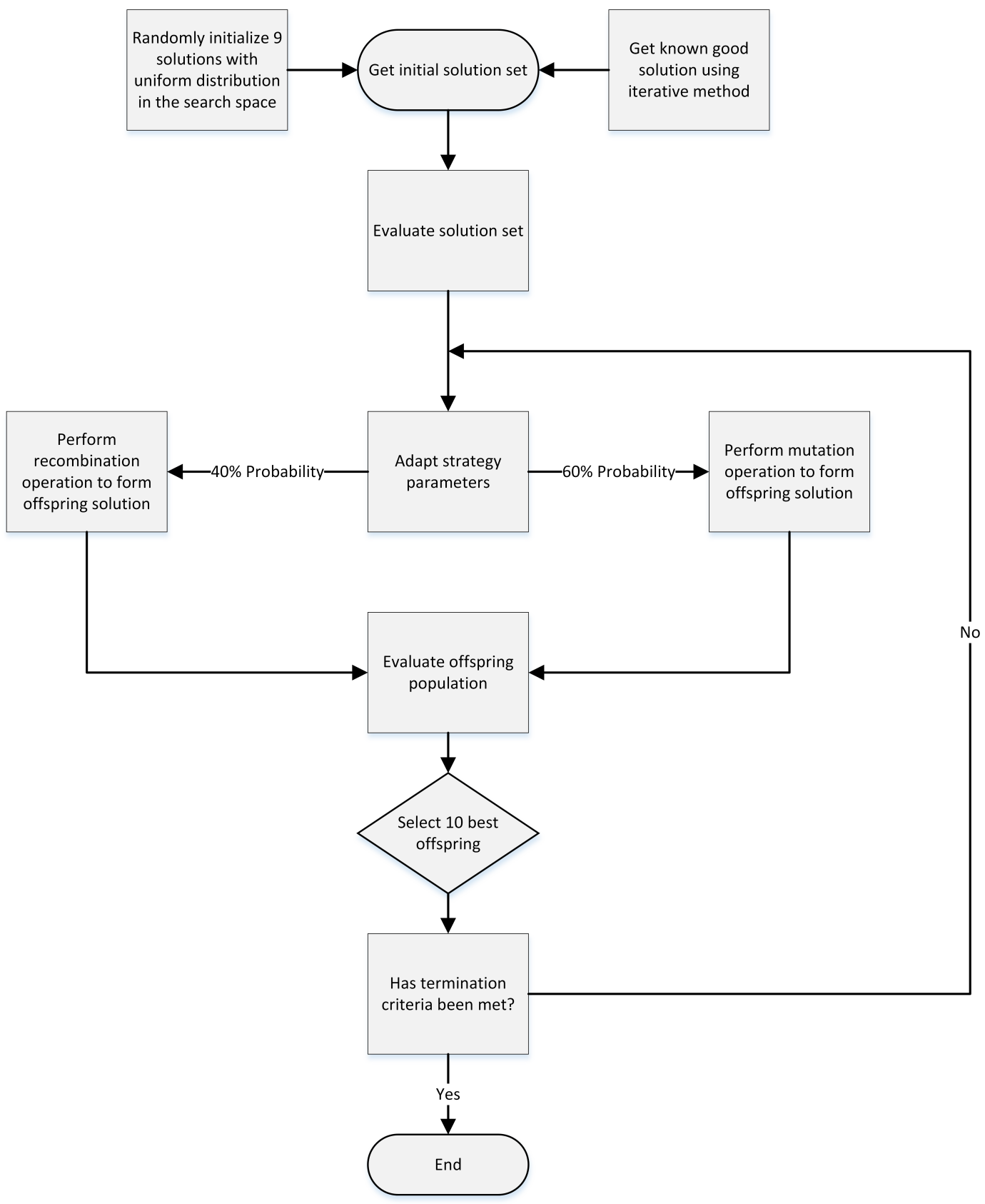

solution in the offspring population, and the next generation begins. This continues until the termination criteria are met. Figure 5.2 provides a flow diagram illustrating the method for the evolution strategy. 
To maintain the emphasis on mutation rather than recombination, the probability of mutation must be greater than $50 \%$. Therefore, values between $60-80 \%$ were tested in 5\% increments over sample January, 2012 data. The truncation ratio, which defines to ratio between the parent and offspring populations, was selected as $1 / 7$, which is on the low end of the typical range of $1 / 7$ to $1 / 2$ for evolution strategies using comma-selection (31). This lower truncation ratio was selected under the hypothesis that having a smaller parent population relative to the offspring population would allow the parent pool to remain within feasible space. This selection was also informed by empirical testing on samples of January 2012 data using ratios of 1/7, 1/5 and 1/2. A compromise was attempted between getting acceptable results and limiting the computation time. There is particular tension between these two objectives in selecting an appropriate population size, setting the number of generations and algorithm runs and performing local search. Again, these values were selected based on runs of 10 generations over sample data from the first and third weeks in January 2012 using parent populations of 10 - 50 in intervals of 10 and evaluating the gains made each generation up to 500 generations over multiple runs using data from January 1, 2012. The final selected algorithm parameters are summarized in Table 5.1.

Table 5.1: Evolution Strategy Parameters

\begin{tabular}{|l|c|}
\hline Parent Population $(\mu)$ & 10 \\
\hline Offspring Population $(\lambda)$ & 70 \\
\hline Parents in Recombination $(\rho)$ & 2 \\
\hline Generations & 25 \\
\hline Runs & 2 \\
\hline Search Depth & 1 \\
\hline Probability of Mutation & $60 \%$ \\
\hline
\end{tabular}


As with the hill-climbing algorithm, the evolution strategy was tested using typical meteorological data for Portland, Oregon during the month of January and Malin LMP data from 2012. When the algorithm was seeded with the known feasible solution, results showed an average improvement over the iterative method of approximately $4.5 \%$, which was significantly more promising than the hill-climbing algorithm. Complete results are presented in Section 6. To provide a further basis for comparison, another approach was sought with a greater emphasis on recombination and self-organization within the population rather than individual evolution via mutation. This led to an exploration of differential evolution.

\subsection{Differential Evolution}

Differential evolution (DE) is a direct, stochastic search method that uses the weighted difference between other individuals in the population to mutate the target vector (32). The mutated vector is then recombined with the original target vector with some probability. Thus the mutation operation is a function of the relationship between selected individuals in the population, eliminating the need for a separate probability distribution and making the scheme totally self-organizing.

The population is again initialized randomly with uniform distribution in the search space, and then the discontinuity between 0 and $20 \%$ of heat pump operation is enforced. Each individual is evaluated, and mutation is performed. The mutation operation used here is of the form "DE/target-to-best/1," which mutates the target vector by added the weighted 
difference between the target and best-so-far vectors and between two randomly selected vectors from the remaining population (14), (33). The form of the mutation operation is given in Equation 5.11.

$$
V_{i}=X_{i}+0.5\left(X_{\text {best }}-X_{i}\right)+0.5\left(X_{r 1}-X_{r 2}\right)
$$

Where $\mathrm{V}_{\mathrm{i}}$ is the mutated vector, $\mathrm{X}_{\mathrm{i}}$ is the vector undergoing mutation, known as the target vector, $\mathrm{X}_{\text {best }}$ is the best solution in the population and $\mathrm{X}_{\mathrm{r} 1}$ and $\mathrm{X}_{\mathrm{r} 2}$ are randomly selected individuals in the population.

A trial vector, $U_{i}$, is then generated through the recombination of the mutated vector, $V_{i}$, and the target vector, $\mathrm{X}_{\mathrm{i}}$. Each element in $\mathrm{U}_{\mathrm{i}}$ is taken from either $\mathrm{V}_{\mathrm{i}}$ or $\mathrm{X}_{\mathrm{i}}$, where the element from $V_{i}$ will be selected with some cross-over probability, CR. Otherwise the original $X_{i}$ element is retained. To ensure that at least one element is crossed-over, a random index is selected at the beginning of recombination, ensuring that an element will definitely come from the mutated vector (14).

Following recombination, each trial vector is evaluated. If the value of the trial vector, $\mathrm{U}_{\mathrm{i}}$, is less than or equal to the target vector, $\mathrm{X}_{\mathrm{i}}$, then $\mathrm{U}_{\mathrm{i}}$ is selected to survive. Otherwise, the original target vector is retained. Local search is then performed using the hill-climbing method described in Section 5.6. Figure 5.3 provides a flow diagram illustrating the method for the differential evolution algorithm.

The literature recommends that the population size for a differential evolution algorithm be several times the number of elements in the individuals (34), (32), (14). However, given 
Figure 5.3: Flow Diagram Describing the Differential Evolution Process

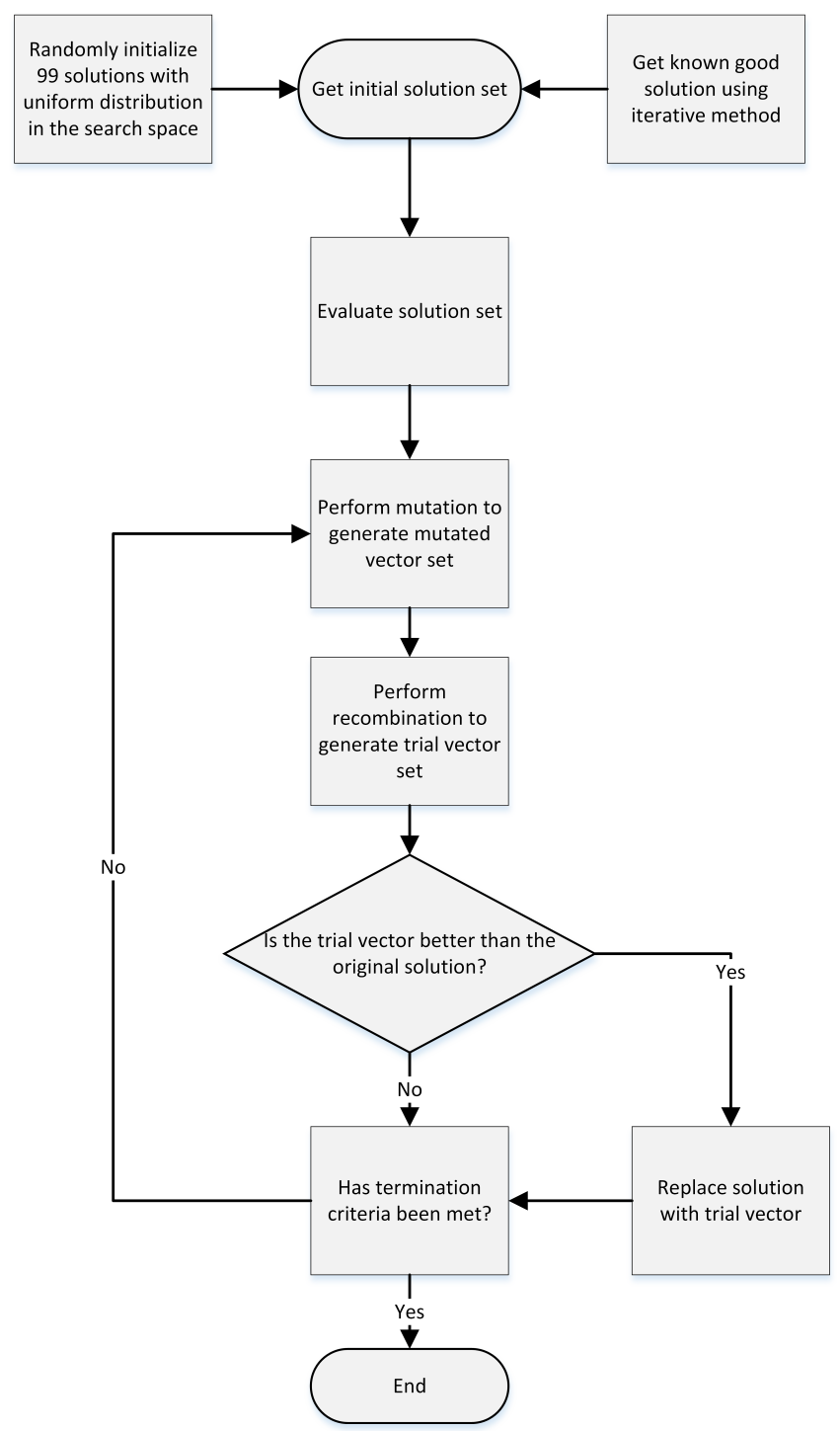


the run-time requirement for this problem, this was simply not practical, and so once again, a compromise had to be made between the population size, the number of generations and the number of runs in order to meet this run-time requirement. The parameters selected for the algorithm are given in Table 5.2.

There is no general consensus on how to appropriately select the cross-over rate for mutation. There has been work done that suggests that smaller values, less than or equal to $20 \%$, are more appropriate for separable functions while larger values, as high as $90 \%$, will allow for a more efficient search of the space, but this work is not definitive (35), (14). To tune the parameter for this application, the empirical tests were run on sample data from the first and third weeks in January 2012 using values between $10 \%$ and $90 \%$ in $10 \%$ increments before $30 \%$ was selected based on these preliminary results.

Table 5.2: Differential Evolution Parameters

\begin{tabular}{|l|c|}
\hline Population & 100 \\
\hline Generations & 10 \\
\hline Runs & 1 \\
\hline Scale Factor (F) & 0.5 \\
\hline Cross-Over Probability (CR) & $30 \%$ \\
\hline Search Depth & 1 \\
\hline
\end{tabular}




\section{Optimization Results}

Average results from six runs of the hill-climbing, evolution strategy and differential evolution algorithms for every day in January 2012 are given in Table 6.4 along with the results from the iterative method that was used in the study described in Section 4. The performance of each of the algorithms was then compared to the performance of iterative method described in Section 3.3 and used in the Scoping Study. The percent improvement for each algorithm is given in Table 6.1, where improvement is defined as a reduction in the utility's wholesale electricity cost to operate the residential heating system. Each algorithm was then run on the first day of January 100 times to find the standard deviation between runs in order to evaluate the amount that the results vary, which is a concern with stochastic optimization methods. These results are presented in Table 6.2.

Table 6.1: Average Algorithm Improvement Over Iterative Method for January 2012

\begin{tabular}{|l|c|}
\hline Algorithm & Improvement (\%) \\
\hline Hill-Climbing & 0.11 \\
\hline Evolution Strategy & 5.0 \\
\hline Differential Evolution & 4.9 \\
\hline
\end{tabular}

Table 6.2: Standard Deviations for January 1, 2012 Over 100 Runs

\begin{tabular}{|l|c|c|}
\hline Algorithm & Std. Dev. & As \% of Mean \\
\hline Evolution Strategy & 0.0044 & 1.5 \\
\hline Differential Evolution & 0.0033 & 1.2 \\
\hline
\end{tabular}


The algorithms were then applied to the month of April in 2014 to evaluate their performance in a second quarter month where load shifting could play a particularly critical role in increasing the utilization factor of variable wind resources. During the spring season, the evolution strategy performed significantly better than differential evolution. While both demonstrated improvement over the iterative method during the four April runs performed, the evolution strategy showed an average improvement of $19.6 \%$, which is roughly four times that seen during winter month tests. This is particularly promising as it indicates the ability to exploit times of very low or negative energy prices, which will realize the load-shifting that the project is intended to accomplish.

However, the algorithm's performance was also less consistent than was seen during winter months with a range of results from a $16.0 \%$ improvement to a $23.2 \%$ improvement over the four runs. This could be mitigated in part by running the algorithm several times for each optimization period and taking the best results. This would increase computation time, and so this solution may or may not be feasible depending on the software environment and hardware used to run the optimization routine. Average results from the April 2014 tests are given in Tables 6.5 and 6.3. The results from differential evolution were more consistent with a difference of only $0.24 \%$ improvement between runs, but the gains were also significantly more modest.

Table 6.3: Average Algorithm Improvement Over Iterative Method for April 2014

\begin{tabular}{|l|c|}
\hline Algorithm & Improvement (\%) \\
\hline Evolution Strategy & 19.6 \\
\hline Differential Evolution & 2.82 \\
\hline
\end{tabular}


The increase in performance improvement from 5\% to $19.6 \%$ for the evolution strategy between the January and April results is likely attributable to the amount of variation in wholesale electricity price that occurs in spring months. In particular, over-generation can cause wholesale electricity prices to become negative in the second quarter. More work would have to be done, however, to verify this interpretation of the results. The differential evolution algorithm underperformed in April relative to both its January performance and the performance of the evolution strategy. Again, more work would need to be done to examine why this phenomenon occurred, but it may be related to a susceptibility of the differential evolution to edge effects, which occur due to handling the optimization periods as discrete days rather than as a rolling window. This is discussed further in Section 7.

Results from testing the evolution strategy during October of 2014 showed significantly less improvement over the iterative method at an average of $0.78 \%$ over 3 runs. This was anticipated as there are fewer gains to be made during autumn months where wholesale electricity prices are neither driven up by peak heating demand periods as occurs in winter months or driven down due to periods of high wind production as seen in spring months. Testing of the algorithm during January of 2013 showed even greater improvement than that was seen over January of 2012 with an average rate of $12 \%$ improvement compared to iterative method. The improved performance in 2013 is likely caused by the greater variation in wholesale prices that occur in January 2013 compared to January 2012. The average price per MWh increases from \$29 in January 2012 to $\$ 31$ in January 2013 and the standard deviation as a percentage of the mean increases from $24 \%$ to $29 \%$. 
Table 6.4: Algorithm Results from January 2012 in Dollars per Residence in Wholesale Electricity Costs

\begin{tabular}{|c|c|c|c|c|}
\hline Day & Iterative Method & Hill-Climbing & Evolution Strategy & Differential Evolution \\
\hline 1 & 0.300 & 0.299 & 0.289 & 0.283 \\
\hline 2 & 0.291 & 0.290 & 0.277 & 0.276 \\
\hline 3 & 0.261 & 0.261 & 0.248 & 0.247 \\
\hline 4 & 0.266 & 0.265 & 0.252 & 0.251 \\
\hline 5 & 0.200 & 0.200 & 0.191 & 0.189 \\
\hline 6 & 0.174 & 0.174 & 0.167 & 0.161 \\
\hline 7 & 0.191 & 0.191 & 0.179 & 0.181 \\
\hline 8 & 0.216 & 0.216 & 0.203 & 0.205 \\
\hline 9 & 0.211 & 0.211 & 0.199 & 0.200 \\
\hline 10 & 0.272 & 0.271 & 0.259 & 0.254 \\
\hline 11 & 0.317 & 0.317 & 0.298 & 0.301 \\
\hline 12 & 0.262 & 0.262 & 0.247 & 0.249 \\
\hline 13 & 0.234 & 0.234 & 0.221 & 0.221 \\
\hline 14 & 0.230 & 0.230 & 0.217 & 0.220 \\
\hline 15 & 0.161 & 0.160 & 0.152 & 0.152 \\
\hline 16 & 0.181 & 0.180 & 0.172 & 0.169 \\
\hline 17 & 0.191 & 0.190 & 0.183 & 0.181 \\
\hline 18 & 0.180 & 0.180 & 0.172 & 0.171 \\
\hline 19 & 0.141 & 0.141 & 0.134 & 0.136 \\
\hline 20 & 0.194 & 0.194 & 0.184 & 0.188 \\
\hline 21 & 0.163 & 0.163 & 0.155 & 0.157 \\
\hline 22 & 0.172 & 0.172 & 0.164 & 0.167 \\
\hline 23 & 0.212 & 0.211 & 0.200 & 0.202 \\
\hline 24 & 0.226 & 0.226 & 0.216 & 0.214 \\
\hline 25 & 0.110 & 0.110 & 0.104 & 0.106 \\
\hline 26 & 0.083 & 0.083 & 0.080 & 0.081 \\
\hline 27 & 0.146 & 0.146 & 0.140 & 0.142 \\
\hline 28 & 0.159 & 0.159 & 0.152 & 0.154 \\
\hline 29 & 0.187 & 0.187 & 0.178 & 0.177 \\
\hline 30 & 0.173 & 0.173 & 0.165 & 0.168 \\
\hline 31 & 0.175 & 0.175 & 0.168 & 0.171 \\
\hline Total & 6.28 & 6.27 & 5.96 & 5.97 \\
\hline
\end{tabular}


Table 6.5: Algorithm Results from April 2014 in Dollars per Residence in Wholesale Electricity Costs

\begin{tabular}{|c|c|c|c|}
\hline Day & Iterative Method & Evolution Strategy & Differential Evolution \\
\hline 1 & 0.130 & 0.129 & 0.129 \\
\hline 2 & 0.104 & 0.104 & 0.104 \\
\hline 3 & 0.0429 & 0.0429 & 0.0429 \\
\hline 4 & 0.0185 & 0.0185 & 0.0185 \\
\hline 5 & 0.0487 & 0.0486 & 0.0487 \\
\hline 6 & 0.0153 & 0.0153 & 0.0153 \\
\hline 7 & -0.0587 & -0.143 & -0.121 \\
\hline 8 & 0.0870 & 0.0766 & 0.0868 \\
\hline 9 & 0.0511 & 0.0413 & 0.0510 \\
\hline 10 & 0.0556 & 0.0484 & 0.0555 \\
\hline 11 & 0.0236 & -0.0334 & 0.0235 \\
\hline 12 & 0.0886 & 0.0735 & 0.0884 \\
\hline 13 & 0.111 & 0.0922 & 0.111 \\
\hline 14 & 0.149 & 0.124 & 0.149 \\
\hline 15 & 0.165 & 0.138 & 0.165 \\
\hline 16 & 0.163 & 0.136 & 0.163 \\
\hline 17 & 0.105 & 0.0878 & 0.105 \\
\hline 18 & 0.0577 & 0.0484 & 0.0576 \\
\hline 19 & 0.0465 & 0.0391 & 0.0465 \\
\hline 20 & 0.133 & 0.112 & 0.133 \\
\hline 21 & 0.108 & 0.0909 & 0.108 \\
\hline 22 & 0.123 & 0.104 & 0.123 \\
\hline 23 & 0.0904 & 0.0764 & 0.0902 \\
\hline 24 & 0.0389 & 0.0330 & 0.0389 \\
\hline 25 & 0.0490 & 0.0415 & 0.0489 \\
\hline 26 & 0.0507 & 0.0431 & 0.0506 \\
\hline 27 & 0.0538 & 0.0458 & 0.0537 \\
\hline 28 & 0.0708 & 0.0603 & 0.0707 \\
\hline 29 & 0.134 & 0.115 & 0.134 \\
\hline 30 & 0.0976 & 0.0835 & 0.0974 \\
\hline Total & 2.35 & 1.89 & 2.29 \\
\hline
\end{tabular}




\section{Discussion}

Assuming an improvement of 5\%, the evolution strategy would reduce utility costs during winter months by roughly $21 \%$. Scaled to 10,000 homes, this would represent a savings of about $\$ 250,000$ per winter. Additionally, the ability to reduce demand during winter peaking events could eliminate the need for investment in costly peaking plants that have low annual utilization factors. Based on the annual life-cycle costs required to meet capacity needs given in PGE's 2013 Integrated Resource Plan, flexible capacity can be valuated at roughly $\$ 210 / \mathrm{kW}$.

In spring months, despite additional gains based on the evolution strategy's performance, the reduction in wholesale electricity costs decreases due to the lower heating demand. Assuming a $19 \%$ improvement over the iterative method, this represents a $30 \%$ reduction in costs. Scaled to 10,000 homes, this yields $\$ 11,000$ in savings. However, the reduction in the cost of wholesale electricity is not the primary benefit that the utility would realize during spring months. The value of the system is in providing flexible capacity to mitigate the economic risk of high wind generation periods in quarter two that coincide with the times when environmental factors limit the curtailment of hydroelectric generation. This will become an increasingly critical issue as Oregon moves toward its goal of meeting 50\% of its electricity needs through renewable energy. 


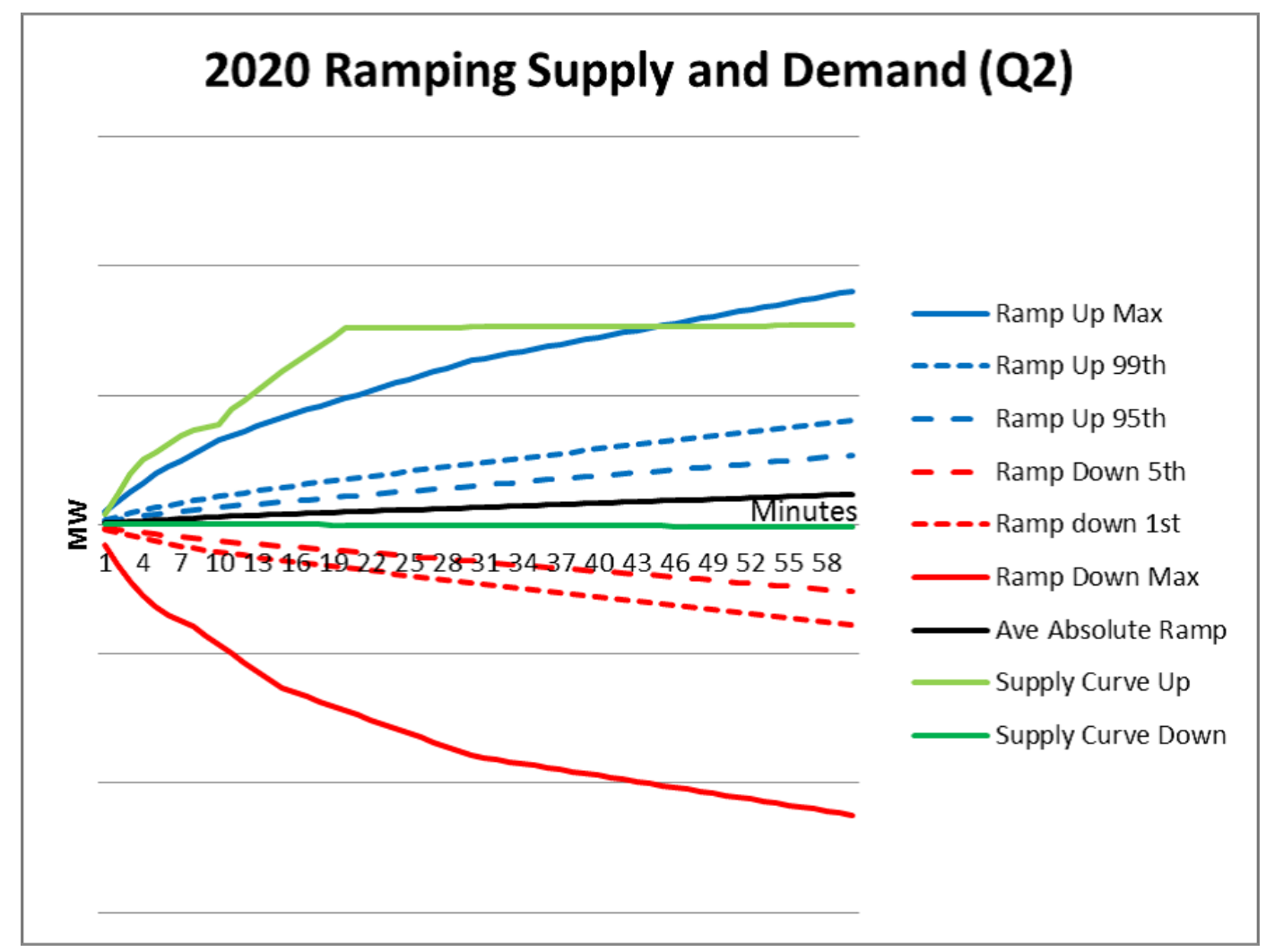

Figure 7.1: PGE 2020 Q2 Supply and Demand for Flexibility

Using the 600 gallon tank, results showed an average daily available energy capacity over the winter and springs months of over $40 \mathrm{kWh}$. Given that the utility-side heat pump is rated at just over $11 \mathrm{~kW}$, this indicates that the system would be able to supply $11 \mathrm{~kW}$ of additional consumption flexibility for more than three hours, which is sufficient to assist in ramping events. This becomes valuable for meeting ramp down requirements, where PGE is vulnerable to deficiency with new wind generation, particularly in the second quarter (Q2). This is demonstrated in Figure 7.1, which was taken from PGE's 2013 Integrated Resource Plan.

With the greater penetration of renewable energy resources, and in particular wind resources, which is being driven by Renewable Portfolio Standards and other regulation, 
PGE is already facing deficiencies in ramp down capability and anticipates that the problem will be further exacerbated moving forward. This is most evident in Q2 projections, where environmental regulation prevents the curtailment of hydro resources during times of high wind generation and low demand. Because wind can be curtailed, ramp down deficiency, unlike ramp up deficiency, is an economic rather than stability risk. However, the potential loss of revenue given wind penetration levels in this region is significant.

The system proposed here, if aggregated, could help reduce these ramp down deficiencies by absorbing some or all of the net difference between electric load and unplanned changes in wind output. Additionally, with enough systems in aggregate and variable speed heat pump operation, the system could further be used for generation following to handle intermittent wind output.

\subsection{Algorithm Performances}

Of the two evolutionary algorithms explored here, the evolution strategy demonstrated the ability to provide more improvement over the first ten minutes of optimization, which is important when the goal is to run the algorithm for only twenty minutes for each period. The differential evolution algorithm performed more slowly but smoothly, providing incremental improvement during every generation rather than the sporadic high bursts of improvement seen by the evolution strategy. The rate of the improvement provided by the evolution strategy decreases more quickly than that of the differential evolution algorithm. Preliminary investigation indicates that, given the ability to run the algorithm for longer, differential 
evolution would provide better results, but more work would have to be done to verify how much improvement this would provide particularly because edge effects seen by the discrete handling of the days seem to impact the differential evolution algorithm's performance more severely.

Figure 7.2 provides an example of the optimization performed by each algorithm for the first day of January 2012, over an hour of algorithm run time on an Intel(R) Core(RM) i72600 CPU Processor. As this demonstrates, the evolution strategy sees greater improvement over the first twenty minutes of operation, but is susceptible to converging on a local minimum. While the algorithm continues to see solution improvement after 40 minutes, the change in solution value is marginal. Increasing the population size of the algorithm would increase its diversity, which would likely help with preventing this premature convergence. However, this would also increase the run time of each generation of the algorithm, which would hinder its early performance. Given the computational power necessary to handle the differential evolution in an acceptable run time, this would be the better choice of algorithm, and this may even be more true when a sliding window is implemented. However, if computational restrictions make running only a limited number of generations possible, as investigated here, the evolution strategy may be the better choice.

\subsection{Limitations and Future Work}

One of the key limitations of this analysis work is that, for the sake of limiting simulation time, the algorithms were evaluated based on discrete 24-hour periods. Each algorithm was 


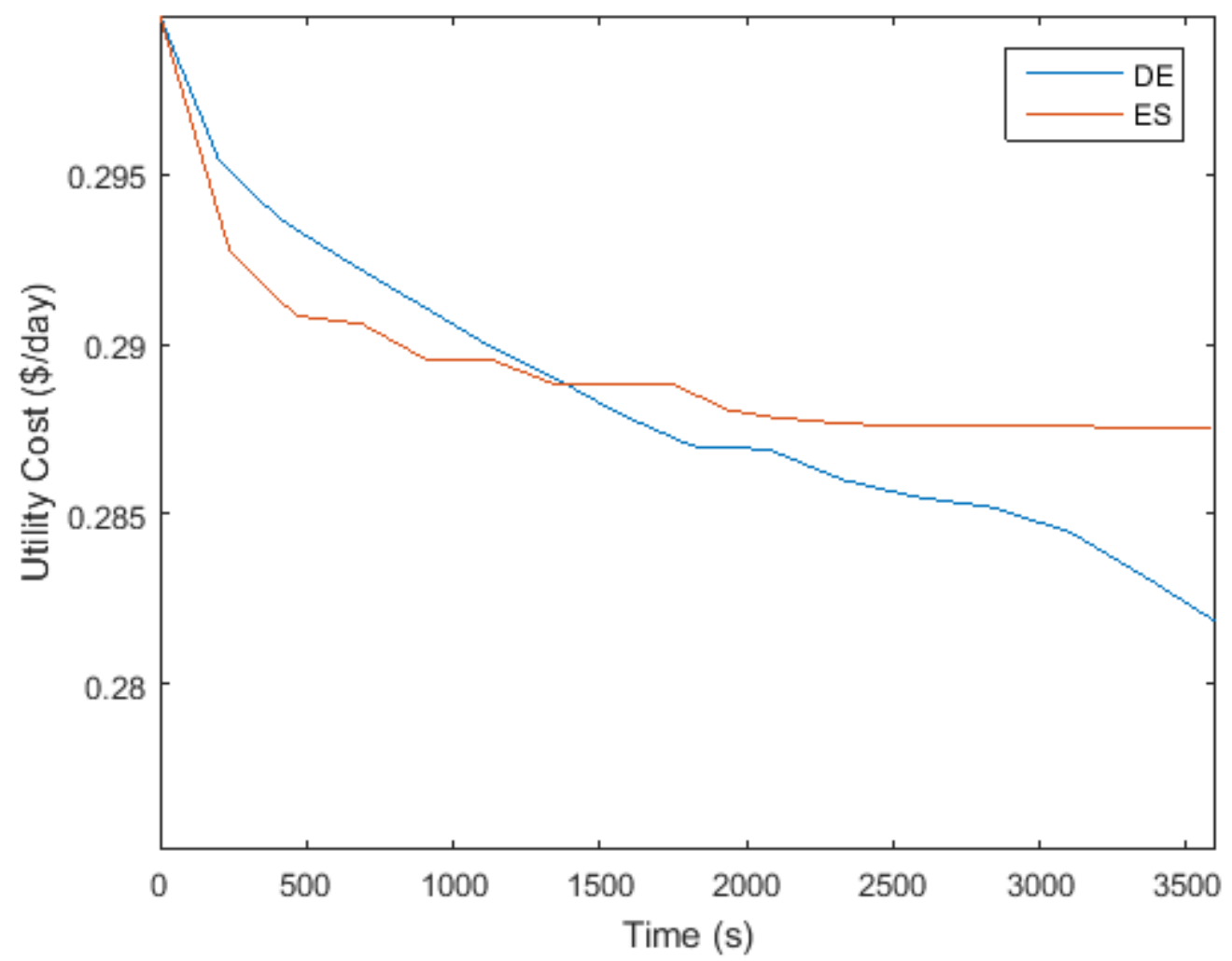

Figure 7.2: Improvement Seen in the Objective for Minimizing Utility Cost for Each Algorithm

run to optimize a day's worth of data in five-minute intervals starting at midnight. Any gains that might be realized in optimizing using a rolling window were therefore sacrificed. Including this in the analysis might impact the relative performances of the two evolutionary algorithms. In general, the evolution strategy performed better over the course a month. However, differential evolution tended to perform better over discrete days with gains of roughly $8 \%$ during winter months rather than the $4 \%$ presented here. This might indicate that operating on a rolling day would stabilize and improve the algorithm's performance by eliminating edge effects. Intuitively, it seems reasonable that further optimization could be performed by both algorithms when using the progressive strategy, but further work is 
required to determine the quantify the actual impact.

Additionally, the system as designed requires that the heat pumps operate outside of their typical operating parameters, and so extrapolation was required to model their performance at some of the boundary conditions. In particular, the specifications for the heat pump used to model the customer unit do not include data for entering water temperatures above $85^{\circ} \mathrm{F}$, which is below the desired maximum operating temperature of $100^{\circ} \mathrm{F}$ described here. Simulations indicated that the system does not typically reach these extreme high temperatures except when set to pre-charge in anticipation of a winter peaking event. To examine the impact of reducing the inlet water temperature to within the rated operating conditions on the peak shaving capability of the system, the simulations were rerun assuming a maximum water temperature of $85^{\circ} \mathrm{F}$, which showed an increase of $0.24 \mathrm{~kW}$, representing a $10 \%$ reduction in peak shaving capacity relative to the benchmark system. Further work needs to be done to create better models of actual heat pump performance under the desired operating conditions using extended empirical test data.

Another concern that has not been addressed here is the sensitivity of both the iterative method used in the Scoping Study and the optimization routines to inaccuracies in the models of the home and heat pumps. In order to operate this system in a home, information on that residence's thermal characteristics as well as specific heat pump specifications would need to be entered to allow the algorithms to run. However, it is probable that these models will be imperfect, and so work needs to be done to determine how detrimental inaccuracies will be to system performance. 


\section{Optimization for the Alpha System Prototype}

\subsection{Alpha Prototype}

The design and construction of an alpha system prototype was included in the scope of the work with which the research team at Portland State was tasked. The prototype was constructed on roughly a $\frac{1}{3}$ scale of the system envisioned and simulated for the preliminary study described previously according to the relative size of the customer-size heat pumps. The water tank was scaled down from the 600-tank given in the prototype to a 55 gallon tank, and the heat pumps were reduced in size from 3 and 2 tons to 1 and 0.75 tons for the utility and customer-sides respectively. Initial testing of the system shows that no meaningful stratification occurs within the tank, which we attribute to its size and the water injection and extraction methods.

The thermal model of the water tank has been modified to reflect its reduced size and the lack of stratification. The rectangular box used to model the customer home was scaled down to roughly $37.5 \%$ of its original size, which assumes a 6,750 cubic foot volume. The heat pump models were similarly reduced in size, though the original COP equations derived from the Daikin and Trane units have been retained. Future work will include the empirical characterization of the actual heat pumps used, which are Climatemaster Tranquility units. Once that occurs, the new COP equations can be incorporated into the structure developed 
for the prototype system optimization routine, which is described in Section 8.2

Due to the decreased size of the system as well as budgetary constraints, non-variable speed heat pumps were selected for the prototype, which requires a different operational scheme. To accommodate this, the optimization routine was modified to handle discrete, on/off heat pump operation. This is fully explained in Section 8.2 below.

\subsection{Optimization for Discrete Heat Pump Operation}

A genetic algorithm was chosen as the strategy for generating operational vectors for the heat pumps. This decision was made because a genetic algorithm is designed to operate on binary strings, which is a convenient mechanism for encoding fixed speed operation where a 0 indicates that the heat pump is off during the time interval in question and a 1 indicates that the heat pump is operating.

An iterative method similar to one the one described in Section 3.3 was created to generate a known feasible solution using discrete heat pump operation. This seeding routine iteratively checks for system constraint violations based on a range of boundary temperature thresholds, and then flips bits within the operational vector to correct detected violations. The lowest cost feasible solution is then.

A discrete hill-climbing algorithm was also developed both as a method of benchmarking the genetic algorithm and as a strategy for local search. A population of fifty 288-bit binary strings is initialized randomly with the exception of one individual, which is the known feasible solution. Similar to the hill-climbing routine described in Section 5.6, each 
individual in the population is then perturbed one element at a time and the fittest of these 288 "neighbor" solutions is selected. This is then repeated twenty times on each individual in the population. Within the context of this algorithm, a neighbor is defined as a binary string having a Hamming Distance of one from the original individual.

The genetic algorithm also begins with forty-nine randomly generated 288-bit binary strings in addition to the known feasible solution, making a total population of fifty. The fitness of each individual is then evaluated and the best individual is saved for elitism. Mutation is then performed with a probability of $2 \%$ using bit-flipping as the mutation operator. This means that probability dictates that an average of 5.76 bits in each 288-bit individual will be flipped during each mutation phase. Once mutation has occurred, the fitness of the mutated individuals is then evaluated, replacing the elite individual if a better solution has been generated.

Two-point cross-over then occurs with a probability of $70 \%$. Once again, fitness is evaluated and local search is performed with a search depth of one on every individual in the population. Finally, a new population is selected probabilistically in proportion to solution fitness. The worst solution in the population is then replaced with the elite solution and the next generation begins. A summary of the algorithm operators and parameters is given in Table 8.1.

The genetic algorithm was tested using typical meteorological data and average hourly locational marginal pricing information from the Malin node in 2012 for January 2012. The genetic algorithm showed a $6 \%$ improvement over hill-climbing, using the modified models 
Table 8.1: Genetic Algorithm Parameters

\begin{tabular}{|l|c|}
\hline Population & 50 \\
\hline Generations & 50 \\
\hline Runs & 2 \\
\hline Mutation Operator & Bit-Flipping \\
\hline Recombination Operator & 2-Point Cross-Over \\
\hline Probability of Mutation & $2 \%$ \\
\hline Probability of Cross-Over & $70 \%$ \\
\hline Local Search Depth & 1 \\
\hline Selection Method & Roulette Wheel \\
\hline
\end{tabular}

described in Section 8.1. While these results may change slightly when the actual COP curves of the heat pumps are incorporated into the algorithm and some of the parameters may need to be tuned accordingly, the structure of the optimization routine should translate well, and this provides a general indication of what we can anticipate the results will be. An example plot of the evolution of the best solution for a day's operation over 50 generations is given in Figure 8.1. 


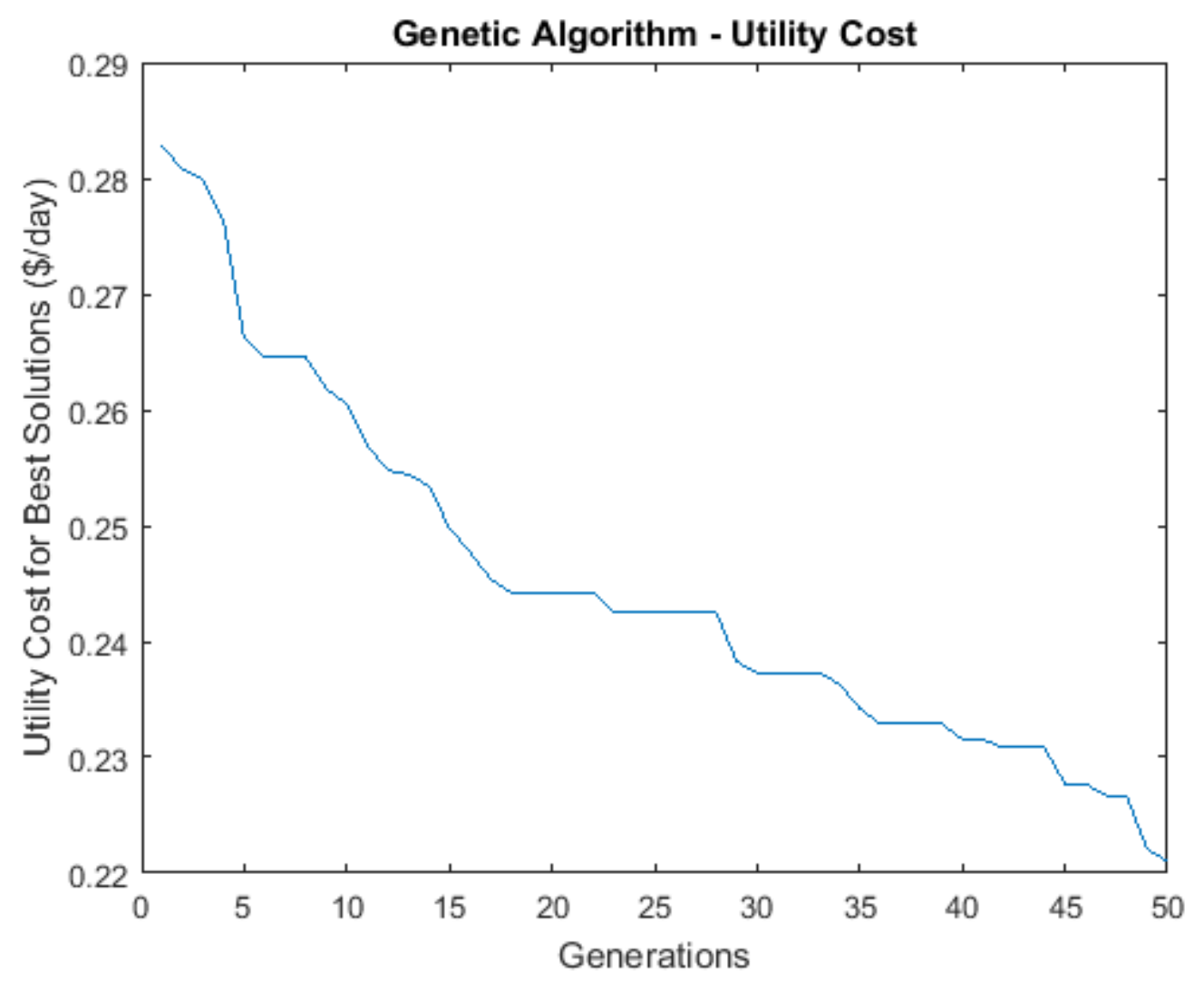

Figure 8.1: Example Plot of Best Solution Evolution 


\section{Conclusion}

The two heat pump system proposed here is capable of realizing a $14 \%$ reduction in the wholesale electricity cost of operating a heating system for a generic single family residence in Portland, Oregon. These savings are based on participation in a non-dispatchable pricebased program informed by real-time pricing. Additional gains can be made by using optimization methods, achieving $20+\%$ improvement during winter months when pricebased programs are most capable of performing peak-shaving in the Portland region. This was established using an evolution strategy that can run in less than an hour to update the operational vector for the utility-side heat pump.

Using active control by the utility, the system could also participate in an event-based or dispatchable program where operators could send command signals to the utility-side heat pump to assist with ramping events or consume excess energy. This becomes particularly valuable to Portland General Electric as the penetration of wind resources on the grid continues to increase. This would help to mitigate the economic risks associated with unplanned wind generation during times of low demand, which are particularly critical during the second quarter where hydroelectric generation cannot be curtailed due to environmental regulation.

Work on the alpha system prototype is on-going, and much remains to be done on fully characterizing that system and integrating the final system models into the genetic 
algorithm that will operate it. As mentioned in Section 7, further work is also warranted in determining the impact on the optimization routine of using a sliding 24-hour window rather than handling each day discretely. Additionally, while the optimization routine was developed and is currently run using Matlab, it will eventually need to be transcribed into a language that can run off of a microprocessor without an expense proprietary license. Work will need to be done to decide what hardware solution is most suitable. Porting the routine into $\mathrm{C}++$ or Python may also have the added benefit of decreasing run times, which would allow for multiple algorithm runs per optimization period thereby decreasing the ultimate variability in algorithm results.

The largest and perhaps most critical future work to be done is in determining whether the project is viable within the market. Cost estimates need to be developed for the system hardware and installation. The project's ultimate feasibility relies heavily on the requisite upfront capital investment by both the utility and the customer. Keeping these costs low will likely depend on the scale of the program and require the engagement of a heat pump manufacturer. The minimum level of penetration necessary to realize meaningful benefits to Portland General Electric has to be estimated and market research needs to be conducted to determine whether enough customer interest can be generated to make these goals attainable. Given that the system as currently proposed has a large footprint and would need to be installed on the customer's premises, it remains to be seen whether the cost savings found in the scoping study would be sufficient to incentivize customer participation or whether additional customer benefits would need to be incorporated into the program. 


\section{Bibliography}

[1] J. Neubauer and M. Simpson, "Deployment of behind-the-meter energy storage for demand charge reduction,” tech. rep., National Renewable Energy Laboratory (NREL), Golden, CO., 2015.

[2] J. P. Barton and D. G. Infield, "Energy storage and its use with intermittent renewable energy," vol. 19, no. 2, pp. 441-448, 2004.

[3] S. A. Pourmousavi, S. N. Patrick, and M. H. Nehrir, "Real-time demand response through aggregate electric water heaters for load shifting and balancing wind generation," vol. 5, no. 2, pp. 769-778, 2014.

[4] N. Beeker, P. Malisani, and N. Petit, "A distributed parameters model for electric hot water tanks," in American Control Conference (ACC), 2015, pp. 4517-4522, 2015.

[5] R. Diao, S. Lu, M. Elizondo, E. Mayhorn, Y. Zhang, and N. Samaan, "Electric water heater modeling and control strategies for demand response," in 2012 IEEE Power and Energy Society General Meeting, pp. 1-8, 2012.

[6] H. Hagiwara, S. Kawachi, J. Baba, K. Furukawa, E. Shimoda, and S. Numata, "Fundamental study on controllability of heat pump power consumption for fluctuated power 
compensation," in 2011 International Conference on Clean Electrical Power (ICCEP), pp. 413-419, 2011.

[7] C. J. C. Williams, J. O. Binder, and T. Kelm, "Demand side management through heat pumps, thermal storage and battery storage to increase local self-consumption and grid compatibility of PV systems," in 2012 3rd IEEE PES International Conference and Exhibition on Innovative Smart Grid Technologies (ISGT Europe), pp. 1-6, 2012.

[8] T. Back, D. B. Fogel, and Z. Michalewicz, Handbook of evolutionary computation. IOP Publishing Ltd., 1997.

[9] T. M. English, "Evolutionary programming vi [book reviews]," Evolutionary Computation, IEEE Transactions on, vol. 1, no. 3, pp. 218-219, 1997.

[10] J. H. Holland, "Outline for a logical theory of adaptive systems," Journal of the ACM (JACM), vol. 9, no. 3, pp. 297-314, 1962.

[11] I. Rechenberg, Evolution Strategy: Optimization of Technical Systems According to the Principles of Biological Evolution. Stuttgart,1 Frommann-Holzboog, 1973.

[12] H.-P. P. Schwefel, Evolution and Optimum Seeking: The Sixth Generation. New York, NY, USA: John Wiley \& Sons, Inc., 1993.

[13] R. Storn and K. Price, Differential evolution-a simple and efficient adaptive scheme for global optimization over continuous spaces, vol. 3. 1995. 
[14] K. Price, R. M. Storn, and J. A. Lampinen, Differential evolution: A Practical Approach to Global Optimization. Springer Science \& Business Media, 2006.

[15] "Thermal model of a house - MATLAB \& simulink example." Online: accessed 29-April-2016.

[16] G. Angrisani, M. Canelli, C. Roselli, and M. Sasso, "Calibration and validation of a thermal energy storage model: Influence on simulation results," vol. 67, no. 1, pp. 190-200, 2014.

[17] "National solar radiation database update - TMY3." Online: accessed 4-June-2015.

[18] "Weather underground." (Online; accessed 5-May-2016).

[19] J. A. C. W. Z. D. DING Qiang, XING Changxin, "Dynamic operational optimization of air source heat pump heating system with consideration of energy saving," CIESC Journal, vol. 66, no. 6, p. 2166, 2015.

[20] J. E. Braun, "Load control using building thermal mass," vol. 125, no. 3, pp. 292-301, 2003.

[21] V. M. Zavala, "Real-time optimization strategies for building systems," Industrial \& Engineering Chemistry Research, vol. 52, no. 9, pp. 3137-3150, 2013.

[22] T. Y. Chen, "Real-time predictive supervisory operation of building thermal systems with thermal mass," vol. 33, no. 2, pp. 141-150, 2001. 
[23] G. Winkler, C. Meisenbach, M. Hable, and P. Meier, "Intelligent energy management of electrical power systems with distributed feeding on the basis of forecasts of demand and generation," in Electricity Distribution, 2001. Part 1: Contributions. CIRED. 16th International Conference and Exhibition on (IEE Conf. Publ No. 482), vol. 4, pp. 5 pp. vol.4-, 2001.

[24] G. Chen, "Improved evolutionary programming \#x00026; its application in economic dispatch problem," in Power and Energy Engineering Conference (APPEEC), 2010 Asia-Pacific, pp. 1-4, 2010.

[25] P. Subbaraj, R. Rengaraj, and S. Salivahanan, "Real-coded genetic algorithm enhanced with self adaptation for solving economic dispatch problem with prohibited operating zone," in 2009 International Conference on Control, Automation, Communication and Energy Conservation, 2009. INCACEC 2009, pp. 1-6, 2009.

[26] T. G. Werner and J. F. Verstege, “An evolution strategy for common unit commitment and economic dispatch of hydrothermal power systems," in International Conference on Electric Power Engineering, 1999. PowerTech Budapest 99, pp. 45-, 1999.

[27] B. Mahdad and K. Srairi, "Differential evolution based dynamic decomposed strategy for solution of large practical economic dispatch,” in 2011 10th International Conference on Environment and Electrical Engineering (EEEIC), pp. 1-5, 2011.

[28] I. Rechenberg, "Evolution strategy: Nature's way of optimization,” in Optimization: Methods and Applications, Possibilities and Limitations (D. H. W. Bergmann, ed.), 
no. 47 in Lecture Notes in Engineering, pp. 106-126, Springer Berlin Heidelberg, 1989. DOI: 10.1007/978-3-642-83814-9_6.

[29] H.-G. Beyer and H.-P. Schwefel, "Evolution strategies - a comprehensive introduction," Natural Computing, vol. 1, no. 1, pp. 3-52.

[30] Y. Matsumura, K. Ohkura, and K. Ueda, "Advantages of global discrete recombination in ( mu;/ mu;, lambda;,)-evolution strategies," in Evolutionary Computation, 2002. CEC'02. Proceedings of the 2002 Congress on, vol. 2, pp. 1848-1853, 2002.

[31] H. Beyer, "Evolution strategies," vol. 2, no. 8, p. 1965, 2007. revision 130731.

[32] R. Storn and K. Price, "Differential evolution - a simple and efficient heuristic for global optimization over continuous spaces," vol. 11, no. 4, pp. 341-359, 1997.

[33] S. Das and P. N. Suganthan, "Differential evolution: A survey of the state-of-the-art," IEEE Transactions on Evolutionary Computation, vol. 15, pp. 4-31, Feb 2011.

[34] A. K. Qin, V. L. Huang, and P. N. Suganthan, "Differential evolution algorithm with strategy adaptation for global numerical optimization," Evolutionary Computation, IEEE Transactions on, vol. 13, no. 2, pp. 398-417, 2009.

[35] J. Ronkkonen, S. Kukkonen, and K. V. Price, "Real-parameter optimization with differential evolution," in The 2005 IEEE Congress on Evolutionary Computation, 2005, vol. 1, pp. 506-513 Vol.1, 2005. 


\section{Appendix A: COP Equations}

\section{A.1 Variable Definitions}

$T_{a}:$ Ambient Temperature $(K)$

$T_{w}:$ Water Temperature $(K)$

$C O P$ : Heat pump coefficient of performance

$\dot{m}$ : Water flow rate $\mathrm{kg} / \mathrm{s}$

\section{A.2 Benchmark Air-to-Air Heat Pump}

The equation for calculating the benchmark heat pump's coefficient of performance as a function of the ambient air temperature was interpolated from the specifications of a 2 ton Carrier Home Comfort heat pump with a HSPF of 8.5 and is given in Equation A.1. The coefficients for space heating mode are given in Table A.1. Note that the coefficients provided here for all three COP equations include all of the digits used in the performing the COP calculations. When fewer digits were used for certain coefficients, the fidelity of the models to the equipment specifications was compromised, and so all of the digits were retained.

$$
C O P_{b}=c_{1} T_{a}^{2}+c_{2} T_{a}+c_{3}
$$


Table A.1: Benchmark Heat Pump COP Coefficients

\begin{tabular}{|l|c|}
\hline$c_{1}$ & 0.001036618737066 \\
\hline$c_{2}$ & -0.487480709965936 \\
\hline$c_{3}$ & 57.795716262660626 \\
\hline
\end{tabular}

\section{A.3 Utility-Side Heat Pump}

The equation for calculating the utility-side heat pump's coefficient of performance as a function of the leaving water temperature and the ambient air temperature was interpolated from the specifications of a 3.2 ton Daikin Air-to-Water heat pump and is given in Equation A.2. The coefficients for water heating and cooling mode are given in Table A.2.

$$
C O P=c_{1} T_{a}^{2} T_{l w}{ }^{2}+c_{2} T_{a}^{2} T_{l w}+c_{3} T_{a}^{2}+c_{4} T_{a} T_{w}{ }^{2}+c_{5} T_{a} T_{w}+c_{6} T_{a}+c_{7} T_{w}{ }^{2}+c_{8} T_{w}+c_{9} \text { (A.2) }
$$

Table A.2: Utility-Side Heat Pump COP Coefficients

\begin{tabular}{|c|c|c|}
\hline Coefficient & Water Heating Mode & Water Cooling Mode \\
\hline$c_{1}$ & -0.0000069799987842105791 & 0.0000097978489006188681 \\
\hline$c_{2}$ & 0.004273691410554629 & -0.0055767805677078043 \\
\hline$c_{3}$ & -0.65199966934276532 & 0.7920974647667981 \\
\hline$c_{4}$ & 0.0040140856414434441 & -0.0059200956542583057 \\
\hline$c_{5}$ & -2.4623917638476516 & 3.3670361713693122 \\
\hline$c_{6}$ & 376.55576426211297 & -477.94850373052077 \\
\hline$c_{7}$ & -0.57565417099277261 & 0.89392549292799683 \\
\hline$c_{8}$ & 353.64815505175926 & -507.94742334385313 \\
\hline$c_{9}$ & -54179.724395432037 & 72051.477720131996 \\
\hline
\end{tabular}

\section{A.4 Customer Heat Pump Coefficient of Performance}

The equation for calculating the customer-side heat pump's coefficient of performance as a function of the entering water temperature and the rate of water flow was interpolated from 
the specifications of a 2 ton Trane Water Source heat pump and is given in Equation A.3.

The coefficients for space heating and cooling mode are given in Table A.3.

$C O P=c_{1} T_{w}{ }^{2} \dot{m}^{2}+c_{2} T_{w}{ }^{2} \dot{m}+c_{3} T_{w}{ }^{2}+c_{4} T_{w} \dot{m}^{2}+c_{5} T_{w} \dot{m}+c_{6} T_{w}+c_{7} \dot{m}^{2}+c_{8} \dot{m}+c_{9}$ (A.3)

Table A.3: Customer-Side Heat Pump COP Coefficients

\begin{tabular}{|c|c|c|}
\hline Coefficient & Space Heating Mode & Space Cooling Mode \\
\hline$c_{1}$ & 0.00003363209211505644 & -0.000064956431154372207 \\
\hline$c_{2}$ & -0.00058992807976689912 & 0.0013942091868543629 \\
\hline$c_{3}$ & 0.0025288244542452285 & 0.0033428015910137413 \\
\hline$c_{4}$ & -0.020350885204577642 & 0.040787283559928389 \\
\hline$c_{5}$ & 0.35367679716917211 & -0.87223178281017888 \\
\hline$c_{6}$ & -1.4171752189122095 & -2.1612403431516269 \\
\hline$c_{7}$ & 3.0652011845207228 & -6.41141062936134 \\
\hline$c_{8}$ & -52.808692972640387 & 136.5465956155237 \\
\hline$c_{9}$ & 203.82815740244629 & 352.17225041204171 \\
\hline
\end{tabular}

